\title{
Universal metric properties of bifurcations of endomorphisms
}

\author{
B Derrida $\dagger$, A Gervois $\ddagger$ and Y Pomeau $\ddagger$ \\ † Institut Laue-Langevin, BP156, 38042 Grenoble Cédex, France \\ $\ddagger$ C.E.A. Centre d'Etudes Nucléaires de Saclay, Division de la Physique, BP2, 91190 \\ Gif-sur-Yvette, France
}

Received 6 March 1978

\begin{abstract}
Endomorphisms of the real axis with one extremum have some universal metric properties which depend only on their analytic dependence near the extremum (bifurcation velocity, reduction parameter). It is shown how this problem is similar to the renormalisation problem, and how the bifurcation velocity may be derived from a fixed-point theory.
\end{abstract}

Several renormalisation methods are discussed for the reduced endomorphism

$$
\mathscr{T}_{a}: x \rightarrow \mathscr{T}_{a}(x)=1-a|x|^{1+\varepsilon} .
$$

They all give the same (exact) result for small $\epsilon$.

\section{Introduction}

Because of their relative simplicity, endomorphisms of the real axis have been extensively studied, and a number of results have been obtained for some classes of functions (May 1976, Li and Yorke 1975).

In this paper, we intend to give some results for endomorphisms (or mappings) $T_{a}$ of an interval $I \subset \mathbb{R}$ depending on one parameter $a$; the function $T_{a}$ is continuous, convex with a unique maximum (or critical point) on $I$. It is convenient to assume that the derivative $(\mathrm{d} / \mathrm{d} x) T_{a}(x)$ exists except perhaps at the critical point.

In most theoretical works which have been devoted to this class of mappings, supplementary conditions have been introduced, but it appears from numerical calculations that they are not essential. Some universal features appear which are independent of the shape of the function $T_{a}$. Roughly, they are of two kinds. Some of them depend only on the existence of a unique maximum and can be called structural, e.g. the order of occurrence of periods (Metropolis et al (1973) and the internal similarity (Derrida et al 1978, referred to hereafter as I). The other universal results may be called metric (Feigenbaum $1977 \mathrm{a}, \mathrm{b}$ ) and depend on the shape of $T_{a}$ near the extremum $c$.

For example, if $\left|T_{a}(x)-T_{a}(c)\right| \sim|x-c|^{1+\epsilon}$, the bifurcation velocity and reduction parameter (to be defined in $\S 2.3$ ) depend on the coefficient $\epsilon$ only. Feigenbaum has already pointed out the connection between this property of universality and the idea of renormalisation group theory in critical phenomena.

We shall focus here on the metric properties. Some theoretical results may be obtained, and an extension of the renormalisation group approach is possible. 
In $\S 2$ we recall some definitions for periods and results obtained by Metropolis et al (1973) and by the authors. In $\$ 3$ we enlarge Feigenbaum's considerations and show how the bifurcation problem can be considered as being similar to the phase transition problem. Critical exponents may be defined, but there are an infinite number of critical points (and exponents). In $\$ 4$ we try to get these exponents by approximate renormalisation methods and compare their rapidity of convergence with the exact (numerical) results. Because of universality, we restrict ourselves to the reduced endomorphism $\mathscr{T}_{a}$ of the interval $I=(-1,+1)$, where $a$ is a parameter:

$$
\mathscr{T}_{a}: x \rightarrow \mathscr{T}_{a}(x)=1-a|x|^{1+\epsilon} .
$$

The piecewise linear mapping $(\epsilon=0)$ is already renormalised, and for small $\epsilon$ all methods give the same first-order expression, which can be expected to be in good agreement with the exact result. For the quadratic case $(\epsilon=1)$ the simplest method gives the exact result within an error of $20 \%$, and more sophisticated ways reach an accuracy better than $1 \%$ with not too much calculation. In $\$ 5$ we derive directly for small $\epsilon$ the first-order correction to the piecewise linear case $\epsilon=0$, and obtain the same results as by renormalisation theory in $\S 4$. Even when we put $\epsilon=1$ (quadratic case) into the first-order formulae, we get the exact result within an error of $2 \%$ !

\section{Generalities on periodic points}

\subsection{Definitions}

A point $x$ belongs to a period $k$ if

$$
T_{a}^{(k)}(x)=x
$$

with

$$
T_{a}^{(l)}(x) \neq x \quad \text { if } l<k
$$

where by definition

$$
T_{a}^{(1)}(x)=T_{a}(x)
$$

and

$$
T_{a}^{(l)}(x)=T_{a}\left(T_{a}^{(l-1)}(x)\right)=x_{l}
$$

is the $l$ th iterate of $x$. The iterates $x\left(=x_{0}=x_{k}\right), x_{1}, \ldots, x_{k-1}$ form a sequence.

The period is stable if for a neighbourhood $U$ of $x_{0}$

$$
y \in U \Rightarrow\left|T_{a}^{(l)}\left(x_{0}\right)-T_{a}^{(l)}(y)\right| \rightarrow 0
$$

for $l$ sufficiently large.

When the derivative $(\mathrm{d} / \mathrm{d} x) T_{a}(x)=T_{a}^{\prime}(x)$ exists, the above condition is equivalent to

$$
-1<s<1
$$

with

$$
s=T_{a}^{\prime}\left(x_{0}\right) T_{a}^{\prime}\left(x_{1}\right) \ldots T_{a}^{\prime}\left(x_{k-1}\right) .
$$

When $a$ increases, a stable period appears for the value $a_{s}$ of parameter $a$ for which 
$s=+1$ and disappears for the value $a_{u}$ of $a$ for which $s=-1$. Then a period $2 k$ becomes stable; $a_{u}$ is by definition a bifurcation point on the parameter axis. Interval $\left(a_{s}, a_{u}\right)$ is the stability zone.

For a given value $a$ of the parameter, three situations may occur: (i) no stable period exists; (ii) one stable period exists; (iii) two or more stable period exist. In the latter case it may happen that two points attracted by two different stable periods may be as near to each other as we like, and many complications occur. Presumably these difficulties may be avoided in general when one restricts oneself to the class of functions defined in the introduction, though Metropolis et al (1973) give an example where two stable periods coexist. At least it is believed that the number of these 'spurious' periods is finite, and we may exhibit endomorphisms where only one stable period exists. By definition a 'spurious' period is not found in the construction of Metropolis et al. This is due to the fact that, when the mapping is continuously changed, the critical point never belongs to this spurious period. For example, the piecewise linear mapping (Derrida and Pomeau 1979)

$$
T_{\lambda}(x)= \begin{cases}\lambda x & x<1 \\ \lambda & 1<x<r \\ \lambda(1+r)-\lambda x & r<x\end{cases}
$$

and its peculiar case $r=1$ that we studied in I have no 'spurious' periods.

In the following, we shall assume that situation (iii) above does not occur.

\subsection{Superstability and MSS sequences}

The period $k$ is superstable if $s=0$. It corresponds to a value of the parameter $a$ for which the critical point belongs to the period. Choosing then $c=x_{1}$, the $(k-1)$ iterates of $c$ may be represented by a formal sequence $Q$ of $(k-1)$ characters $R$ (right) or $L$ (left)

where

$$
Q=\sigma_{1} \ldots \sigma_{k-1}
$$

$$
\sigma_{i}=\left\{\begin{array} { l l } 
{ R } & { \text { if } }
\end{array} \quad T _ { a } ^ { ( i ) } ( c ) \left\{\begin{array}{l}
>c \\
L
\end{array} .\right.\right.
$$

For example, the fixed point is represented by a sequence with 0 character (the 'blank' sequence $b$ ), the period 2 by the only character $R$, and so on.

All sequences of $R$ and $L$ characters do not represent a period. For example, the first two symbols are always $R L$. When a sequence $Q$ of $(k-1)$ characters represents an actual superstable period $k$, we say that the sequence $Q$ is allowed. Its length is $k-1$. For $k=5$, we have only three allowed sequences: $R L^{3}, R L^{2} R$ and $R L R^{2}$.

These sequences may be visualised by setting on an axis the iterates $x_{1}, x_{2}, \ldots$ of the extremum (see figure 1). When $a$ goes from $a_{s}$ (beginning of stability) to $a_{u}$ (end of stability) and to larger values passing through the superstable value, the ordering of the iterates is unchanged. Superstable periods give then a good picture of the whole stability zone, hence their importance. We shall use them indifferently to denote either the whole corresponding stability zone or the point of superstability in the parameter space. Except for periods 1,2 and 3, many sequences exist corresponding to periods with the same number of points. The ordering of the point of the period and the location of the stability zone in the parameter space depend on the sequence. This is shown for periods with five points on figure 1 . 

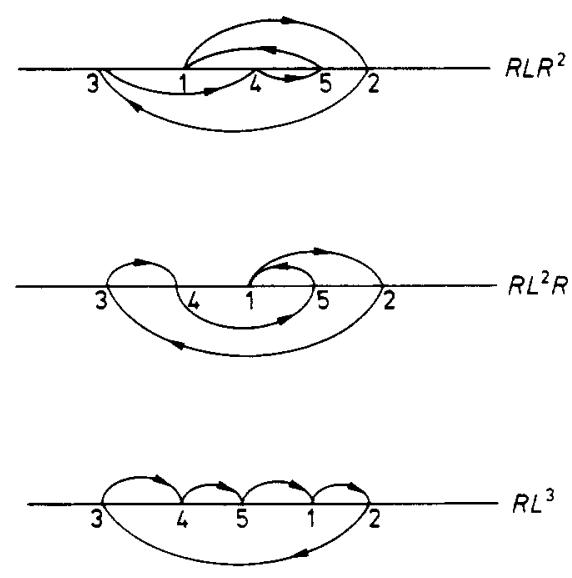

Stability zones

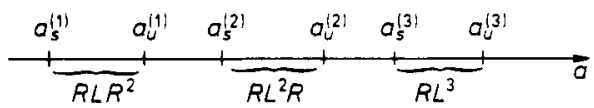

Figure 1. Graphic representation of the iterates of the critical point 1 on an axis for the three allowed sequences with period 5 . The last line shows for these sequences the nonintersecting stability zones on the parameter axis.

These sequences $Q$ have been studied by Metropolis et al (1973) and by the present authors in I. We shall call them MSS sequences. We recall here the results which will be useful later on. From now on, we shall denote as $a_{P}, a_{Q}, \ldots$ the value of the parameter for which the periods $P, Q, \ldots$ are superstable.

(i) If $P$ and $Q$ are allowed sequences which are superstable for the values $a_{P}$ and $a_{Q}$ respectively of the parameter, we say that

$$
P<Q \quad \text { whenever } a_{P}<a_{Q} \text {. }
$$

When $a$ increases, the MSS sequences always appear in the same order, independently of $T_{a}$ (universal sequences). For example,

$$
b<R<R L R<R L R^{3} L R<\ldots<R L<R L^{2} R L<\ldots
$$

and

$$
R L^{n_{1}}<R L^{n_{2}} \quad \text { if } n_{1}<n_{2} .
$$

Notice that, as shown in the above example, the first sequence after a given sequence is its harmonic: for instance $R L R$ (period 4) follows $R$ (period 2), $R L^{2} R L$ (period 6) follows $R L$ (period 3), and so on ....

Between two given sequences $P_{1}$ and $P_{2}$, we may always construct the sequence $P_{3}$ of minimal length. Thus the construction and ordering of all MSS sequences is possible (Metropolis et al 1973).

(ii) One may define on the set of allowed sequences an inner composition law denoted by *. $Q$ and $P$ being two allowed sequences corresponding to periods $k$ and $l$, $Q * P$ is an allowed sequence of period $k l$. More precisely, if $P=$ $\sigma_{1} \sigma_{2} \ldots \sigma_{l-1}\left(\sigma_{i}=R, L\right)$,

$$
Q * P=Q \tau_{1} Q \tau_{2} \ldots Q \tau_{l-1} Q
$$


where

$$
\tau_{i} \begin{cases}=\sigma_{i} & \text { if } Q \text { has an even number of } R \text { characters } \\ \neq \sigma_{i} & \text { otherwise }\end{cases}
$$

For example, the harmonic of $Q$ is $Q * R$.

By construction, the law $*$ is not commutative, but is associative with neutral element $b$ (corresponding to period 1). The sequence $Q * P(Q \neq b)$ is said to be factorisable or non-primary. The number of non-primary sequences is small compared with the number of primary sequences (Derrida et al 1977, 1978).

One important consequence is the law of internal similarity. For given $Q$, the mapping $Q *: P \rightarrow Q * P$ maps the whole set of MSS sequences into one of its parts and preserves the ordering relation. The set of all sequences between $b$ and $R L^{N}$ (for every $N$ ) has the same structure as the set of sequences between $Q$ and $Q * R L^{N}$, and conversely every $Q^{\prime}\left(Q<Q^{\prime}<Q * R L^{N}\right)$ can be written in the form $Q^{\prime}=Q * P$. This result is to be related to the Sarkovskii theorem (Sarkovskii 1964, Stefan 1977, Cosnard and Eberhard 1977) and is of great importance in showing the connection with renormalisation. A similar property does not exist for the mapping $* P, P: Q \rightarrow Q * P$, so we shall not deal with it any further.

With the graphic representation, it is easy to see what the non-primary sequences correspond to. Let us show it in an example. For $k=6$, there exist two non-primary sequences $P_{1}=R * R L=R L R^{3}$ and $P_{2}=R L * R=R L^{2} R L$ which are represented in figure 2. For $P_{1}$ (figure $2 a$ ) we may draw a limit between points of the period numbered 5 and 4 ; by mapping $T_{a}$, at every iteration, this limit is crossed. On a large scale-i.e. if one assimilates each of the clusters $1-3-5$ and $4-6-2$ to a single point-the dynamics is that of period 2; on a small scale-i.e. in each of the clusters $1-3-5$ and 4-6-2-the dynamics is that of period 3 . For sequence $P_{2}$, there are two separations (figure $2 b$ ); on a large scale the dynamics is that of period 3, and on a small scale that of period 2. More generally, if $P=P_{1} * \ldots * P_{n}$, there are $n$ successive scales. For instance, if $P=$ $R * R L * R$ (figure $2 c$ ), the dynamics at large, medium and small scale is that of period 2,3 and 2 respectively.

(a)

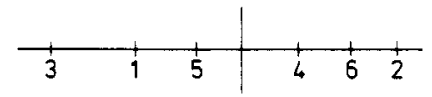

(b)

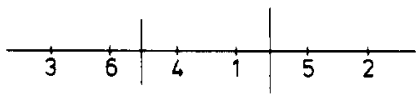

(c)

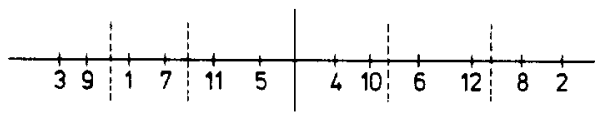

Figure 2. Different scales in non-primary sequences: (a) $P_{1}=R * R L ;(b) P_{2}=R L * R$; (c) $P=R * R L * R$. 
(iii) It is possible to recognise whether a given sequence is allowed or not and to compare it-in the sense of (2.6)-with other sequences without constructing all sequences with a smaller period. The classification has been found-because of the universality property (i)—by studying the simplest function $T_{a}$, i.e. the piecewise linear mapping $\mathscr{L}_{a}$

$$
\mathscr{L}_{a}: x \rightarrow \mathscr{L}_{a}(x)=1-a|x|
$$

(with $a \geqslant 1$, as no superstable period exists if $a<1$ ).

The derivative of $\mathscr{L}_{a}$ does not exist at $x=1$, but the only difference with everywhere derivable endomorphisms $T_{a}$ is that some non-primary periods cancel out.

We shall use in $\S 5$ some results concerning the piecewise linear mapping $\mathscr{L}_{a}$ which have been derived in $\mathrm{I}$. We recall them here:

(a) The value $\bar{a}_{O}$ of the parameter $a$ for which the sequence $Q$ is superstable is the largest real root of the polynomial

$$
\mathscr{P}_{Q}(x)=1+\alpha_{k-2} x+\ldots+\alpha_{k-2} \ldots \alpha_{i} x^{k-1-t}+\ldots+\alpha_{k-2} \ldots \alpha_{0} x^{k-1}
$$

where

$$
\alpha_{i}=-\operatorname{sgn} \mathscr{L}_{a}^{(i+1)}(0)
$$

and

$$
\operatorname{sgn} x= \begin{cases}x /|x| & \text { if } x \neq 0 \\ 0 & \text { otherwise. }\end{cases}
$$

The $\left\{\alpha_{i}\right\}$ are related in an obvious way to the $R$ and $L$ symbols.

(b) Because of the identity proved in I

$$
\mathscr{P}_{Q * P}(x)=\mathscr{P}_{Q}(x) \mathscr{P}_{P}\left(x^{k}\right)
$$

all sequences $Q * P, Q$ being primary, define the same parameter $\bar{a}_{Q}$ except when $Q=R$. The parameter $\bar{a}_{R * P}$ (when $Q=R$ ) is $\bar{a}_{P}^{1 / 2}$.

Notice that, as $\left|(\mathrm{d} / \mathrm{d} x) \mathscr{L}_{a}(x)\right|=a$ except at the critical point, no period is stable except the fixed point at $a<1$.

\section{Metric universal properties $\dagger$}

\subsection{Feigenbaum results}

Feigenbaum $(1977 \mathrm{a}, \mathrm{b})$ succeeded in calculating numerically the value $a_{n}$ of the parameter $a$ of the superstable harmonics $R^{* n}$ (of period $2^{n}$ ) for several functions $T_{a}$. He showed that:

(i) When $n$ increases, the $\left\{a_{n}\right\}$ are an increasing sequence with limit $a_{*}$ and

$$
a_{*}-a_{n} \sim \delta_{R}^{-n} \text {. }
$$

\footnotetext{
$\dagger$ In the bulk of the paper, the word 'critical' appears with two different meanings. In $\S \S 1,2$ and 6 , a critical point for a mapping is a point where the Jacobian of the transformation vanishes (in one-dimensional mappings the point where the derivative $(\mathrm{d} / \mathrm{d} x) T_{a}(x)$ is zero). In $\$ \S 3-5$, critical points and critical exponents refer to the notion of critical points and critical exponents in phase transitions. These two definitions are traditional, and we maintain them since they do not appear at the same time in the paper. We hope that no ambiguity is possible.
} 
More precisely, the ratio

$$
\delta_{n}=\left(a_{n}-a_{n-1}\right) /\left(a_{n+1}-a_{n}\right)
$$

has a limit $\delta_{R}$ (defined by equation (3.1)) when $n$ goes to infinity, and it depends only on the shape near the extremum $c$ (and not on the function). If

$$
\left|T_{a}(x)-T_{a}(c)\right| \sim|x-c|^{1+\epsilon}
$$

$\delta_{R}$ depends on $\epsilon$ only. We shall call $\delta_{R}$ the bifurcation velocity.

(ii) For large $n$, we have the asymptotic conjugation law

$$
T_{a}^{2^{n}}(x) \underset{n \rightarrow \infty}{\sim} \gamma_{R}^{-n} \bar{\varphi}\left(\gamma_{R}^{n} x\right)
$$

where $\bar{\varphi}$ is some (complicated) universal function, and $\gamma_{R}$ is the reduction parameter. Both $\bar{\varphi}$ (up to a linear homomorphism) and $\gamma_{R}$ do not depend on the details of $T_{a}$ but only on the exponent $\epsilon$. The renormalisation methods start from equation (3.4).

For $\epsilon=1$ and different mappings $T$, Feigenbaum found $\delta_{R}=4.66920160903 \ldots$, $\gamma_{R}=2 \cdot 5029078750957 \ldots$

\subsection{Other numerical results}

We repeated the calculations for more general sequences $P_{1} * Q^{* n} * P_{2}$ and corresponding periods $p_{1} q^{n} p_{2}$. We focused our attention on the parameter $a_{P_{1} * Q^{* n} * P_{2}}$ in the particular transformation $\mathscr{T}_{a}$ for several possible sequences $Q$ and $P_{1}$. Actually, for simplicity, we considered only sequences corresponding to small periods, i.e.

$$
\begin{array}{ll}
\text { (a) } Q=R, & P_{1}=b, R L, R L^{2} \\
\text { (b) } Q=R L, & P_{1}=b, R, R L^{2}
\end{array}
$$

and the case $P_{2}=R L^{\infty}$, where $R L^{\infty}$ is the limit for sequences $R L^{n}$ when $n$ is sufficiently large.

Though sequences $R^{* n}$ behave in a slightly different manner than sequences $Q^{* n}$, from our results (see tables 1 and 2) it seems that properties (i) and (ii) of Feigenbaum can be extended in the following way:

(i) The parameter

$$
a_{n}=a_{P_{1} * Q^{n} * P_{2}}
$$

and the ratio

$$
\delta_{n}=\left(a_{n}-a_{n-1}\right) /\left(a_{n+1}-a_{n}\right)
$$

tend to limits $a_{*}$ and $\delta_{Q}$ respectively as $n \rightarrow \infty$. The limit value $\delta_{Q}$ of $\delta_{n}$ is a universal number which depends on $Q$ and $\epsilon$ only.

(ii) When $n$ becomes large, the asymptotic conjugation law (3.4) is true, $\gamma$ and $\bar{\varphi}$ depending only on $\epsilon$ and $Q$. Sequence $P_{2}$ plays no role. This seems reasonable as, when $n$ becomes large, the $p_{1} q^{n}$ first characters of $P_{1} * Q^{* n} * P_{2}$ depend on $Q$ and $P_{1}$ only. The case $\epsilon=1$ and $Q=R(P=b)$ is the one studied by Feigenbaum; for $Q=R L$ we find $\delta_{R L}=55 \cdot 247 \ldots, \gamma_{R L}=9 \cdot 27738 \ldots$ The parameters $\gamma_{R L}$ and $\delta_{R L}$ are much greater than $\gamma_{R}$ and $\delta_{R}$. We shall see in $\S 5$ that this can be explained by computing $\delta_{R L}$ (and more generally $\delta_{Q}$ ) by expansion near $\epsilon=0$. 
Table 1. Fixed-point and bifurcation velocity for the set of sequences $P * Q^{* n}(n \rightarrow \infty)$ for several MSS sequences $Q$ and $P$ in the quadratic case $(\epsilon=1)$ obtained numerically: (a) $Q=R ;(b) Q=R L$.

\begin{tabular}{llllll}
\hline \multirow{2}{*}{$\begin{array}{l}\text { Sequence } \\
P\end{array}$} & (a) $Q=R, \epsilon=1$ & & (b) $Q=R L, \epsilon=1$ & \\
\cline { 2 - 3 } & $a_{*}$ & $\delta_{R}^{-1}$ & $a_{*}$ & $\delta_{R L}^{-1}$ \\
\hline$b$ & $1.40115518909 \ldots$ & $0.21416937 \ldots$ & 1.786440255 & 0.0181005 \\
$(a) R L,(b) R$ & 1.77981807 & 0.2141693 & 1.48458330 & 0.018100 \\
$R L^{2}$ & 1.942173796 & 0.214169 & 1.942550324 & 0.018100 \\
\hline
\end{tabular}

Table 2. Fixed-point and bifurcation velocity for the set of sequences $R^{* n}$ for different values of $\epsilon$. For $\epsilon \leqslant 1$, we have plotted the values we get by using the 'first-order' approximation in $\epsilon$ (see $\$ 5$ ).

\begin{tabular}{|c|c|c|c|c|c|}
\hline \multirow[b]{2}{*}{$\epsilon$} & \multicolumn{2}{|c|}{ Exact calculation } & \multicolumn{3}{|c|}{ Methods $\epsilon \simeq 0$} \\
\hline & $a_{*}$ & $\delta_{R}^{-1}$ & $a_{*}$ & $\left(2+\frac{\epsilon}{\varphi_{*}}\right)^{-1}$ & $\begin{array}{c}\text { for } \epsilon \leqslant 1 \\
\varphi_{*}=a_{*}-1\end{array}$ \\
\hline 0 & 1 & $0 \cdot 5$ & 1 & 0.5 & \\
\hline $0 \cdot 1$ & $1 \cdot 1236$ & $0 \cdot 350$ & $1 \cdot 116$ & 0.349 & \\
\hline 1 & 1.40115 & $0 \cdot 21417$ & $1 \cdot 28$ & 0.179 & \\
\hline 2 & 1.5192995 & $0 \cdot 167$ & & & \\
\hline 5 & 1.68326 & $0 \cdot 10753$ & & & \\
\hline 9 & 1.7726435 & 0.0808 & & & \\
\hline 99 & 1.959263548 & 0.035 & & & \\
\hline
\end{tabular}

\section{Remarks}

1. If $\epsilon=0$ and $Q=R, \delta_{R}=2$ (as $a_{Q^{* n} * P_{2}}=a_{P}^{1 / 2^{n}}$ ).

2. From definition (3.4), $\forall \epsilon$, if $Q=P * P$ then $\delta_{Q}=\delta_{P}^{2}$, and more generally $\delta_{P^{* n}}=$ $\left(\delta_{P}\right)^{n}$, but if $Q=P_{1} * P_{2}$, in general $\delta_{P_{1} * P_{2}} \neq \delta_{P_{1}} \delta_{P_{2}}$. Equality is true at the lowest order when $\epsilon$ is small (and $P_{i} \neq R$ ). When $\epsilon$ is not small, we have the weaker property $\delta_{P_{1} * P_{2}}=\delta_{P_{2} * P_{1}}$. We shall prove it in $\S 3.4$.

\section{3. 'Critical' exponent $\nu_{O}$}

One problem in bifurcation theory is to determine the stability zone for all periods, or equivalently to plot the length of the stable period $k$ as a function of the parameter $a: k=k(a)$. From $\S 2.1$ it is assumed to be unique for a given $a$. When no stable finite period exists, one may set $k(a)=\infty$, so that $k(a)$ is a step function with an infinite number of jumps. For example, the first periods when $a$ increases are the harmonics $R^{* n}$; the stability zones are adjacent and become smaller when $n$ becomes large (see figure $3 a$ ). Setting $a_{n}=a_{R^{* n}}$ for the superstable value of the parameter as $a_{*}-a_{n}$ $\delta_{R}^{-n}$, we have on the $n$th step

$$
k(a)=k\left(a_{n}\right) \sim\left(a_{*}-a_{n}\right)^{\nu_{R}}
$$

which diverges as a power at the limit value $a_{*}$, or more precisely

$$
k(a)=\left(a_{*}-a\right)^{\nu} g_{R}\left(\ln \left(a_{*}-a\right) / \ln \delta_{R}\right)
$$




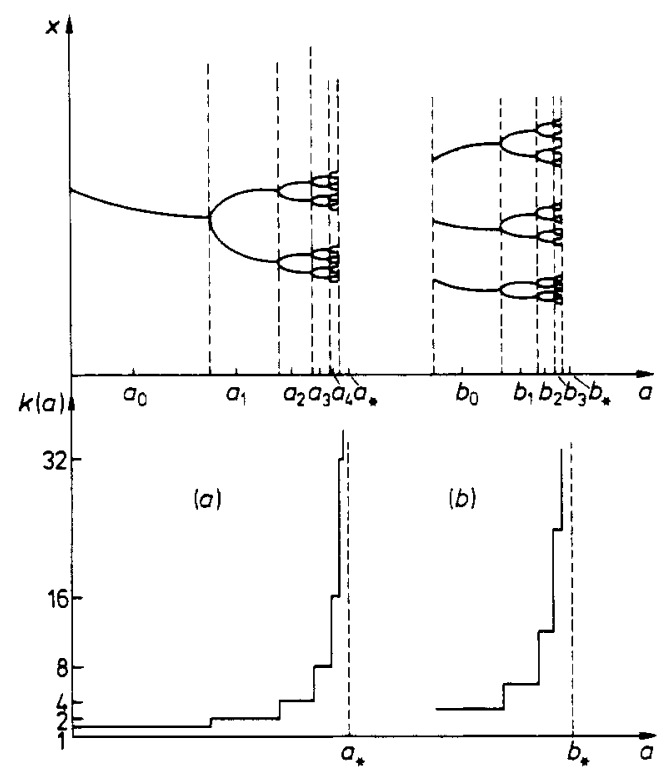

Figure 3. Study of the bifurcation points for the harmonics (a) $R^{* n}$ and (b) $P * R^{* n}$ of period $q$. (For convenience, we took $P=R L, q=3$.) Stability zones are adjacent. The upper figures plot the stable periods as a function of $a$. The superstable values of the parameter $a_{i}=a_{R^{* 1}}$ and $b_{1}=a_{P * R^{* 1}}$ are shown on the $a$ axis. The lower curves show the length of the period as a function of $a ; a_{*}$ and $b_{*}$ are the limit parameters, and the period is multiplied by 2 at each harmonic.

where $g_{R}(x)$ is some discontinuous but periodic function: $g_{R}(x+1)=g_{R}(x)$. The exponent $\nu_{R}$ depends on $R$ and $\epsilon$ only. As $k\left(a_{n+1}\right)=2 k\left(a_{n}\right)$, we get for the critical exponent $\nu_{R}$

$$
\nu_{R}=-\ln 2 / \ln \delta_{R}
$$

For example, if $\epsilon=1, \nu_{R}=-0.449807$.

The same occurs if we consider the harmonics $P * R^{* n}$ of the sequence $P$ of period $p$ (figure $3 b$, with $P=R L$ ); setting $b_{n}=a_{P * R^{* n}}$ as $b_{*}-b_{n} \sim \delta_{R}^{-n}$ and $k\left(b_{n+1}\right)=2 k\left(b_{n}\right)$, we get the same power law $k(b) \sim\left(b_{*}-b_{n}\right)^{\nu_{R}}$, where $\nu_{R}$ is again given by (3.7).

The situation is more complicated if we consider the sequences $R^{* n} * P$, though the limit point is $a_{*}$, as for $R^{* n}$, and periods are multiplied by a factor of 2 . The stability zones are no longer adjacent, and between each zone there is a complicated structure with an infinite number of discontinuities. Because of internal similarity, the fine structure between $c_{n}=a_{R^{* n}{ }_{* P}}$ and $c_{n-1}$ is reproduced between $c_{n+1}$ and $c_{n}$ once the periods are multiplied by 2 . A schematic plot of this situation can be found in figure 4 . If again for the superstable values $c_{n}$ of the parameter we have the power law $k\left(c_{n}\right) \sim$ $\left(c_{n}-a_{*}\right)^{\nu_{R}}$, the true function $k(a)$ is of the form

$$
k(a) \sim\left(a-a_{*}\right)^{\nu} g_{R}\left(\ln \left(a-a_{*}\right) / \ln \delta_{R}\right)
$$

where $g_{R}$ is periodic $\left(g_{R}(x+1)=g_{R}(x)\right)$ and has, in general, an infinite number of jumps. The above expression describes the behaviour of $k(a)$ near $a_{*}$, even for values of $a$ which do not correspond to a superstable period. 


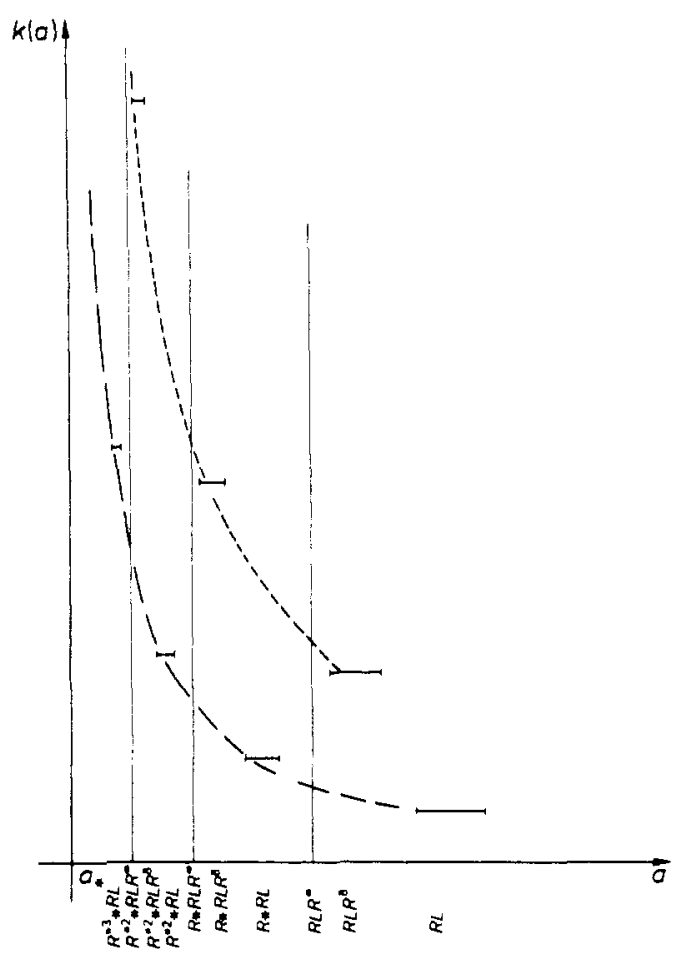

Figure 4. Plot of $R^{* n} * R L, R^{* n} * R L R^{\infty}$ and $R^{* n} * R L R^{8}$ when $a \rightarrow a_{*}\left(a>a_{*}\right)$. The same patterns (multiplied by a factor of 2 ) may be found for each $n$, especially the infinite value of $k$ for $R^{* n} * R L R^{\infty}$. The power law (3.6) is true for $P=R L(---)$ and $P=R L R^{8}$ (- - separately. For convenience, the scale is not respected.

More generally, we may consider all sequences $P_{1} * Q^{* n} * P_{2}$, where $Q \neq R$ is some sequence of period $q$. All the superstable parameters $a_{n}=a_{P_{1} * Q^{* n} * P_{2}}$ converge to a limit $a *=a_{P_{1} * Q^{* \infty}}\left(a_{*}\right.$ depending on $P_{1}$ but not on $\left.P_{2}\right)$, with velocity $\delta_{Q}$, and periods are multiplied by $q$. Again the stability zones are not adjacent; infinitely many discontinuities exist between them. But for superstable periods

$$
k\left(a_{n}\right) \sim\left|a_{*}-a_{n}\right|^{\nu_{0}}
$$

where $\nu_{Q}$ is given by

$$
\nu_{Q}=-\ln q / \ln \delta_{Q}
$$

(if $\epsilon=1$ and $Q=R L$, we get $\nu_{Q}=-0.27384$ ), and near $a_{*}$, we take into account all jumps and infinite discontinuities by writing

$$
k(a) \sim\left|a_{*}-a\right|^{\nu} \circ g_{Q}\left(\ln \left|a_{*}-a\right| / \ln \delta_{O}\right)
$$

where $g_{Q}$ is some complicated function which is periodic $\left(g_{Q}(x+1)=g_{Q}(x)\right)$ and may be infinite for certain values of its argument.

\section{Remarks}

1. The function $g_{Q}$ is not the same for $a<a_{*}$ and $a>a_{*}$. This can be seen in figures $3(a)$ and 4 for $Q=R$. 
2. From (3.9) and (3.5), $\nu_{Q^{* 1}}=\nu_{Q}$, but there is no simple relation between $\nu_{Q_{1} * Q_{2}}, \nu_{Q_{1}}$ and $\nu_{Q_{2}}$. Nevertheless, as $\delta_{Q_{1} * Q_{2}}=\delta_{Q_{2} * Q_{1}}$, we have the weaker result $\nu_{Q_{1} * Q_{2}}=\nu_{O_{2} * Q_{1}}$.

\subsection{Theoretical background}

If $P$ and $Q$ are MSS sequences, the parameter $a_{Q * P}$ corresponding to the superstability of the non-primary MSS sequence $Q * P$ is related to $a_{P}$ by a function of the form

$$
a_{Q * P}=f_{Q}\left(a_{P}\right)
$$

where $f_{Q}$ is monotonic with respect to $P$ (and thus to $a_{P}$ ). We may calculate $f_{Q}$ by expansion near $\epsilon=0$ around the broken linear case (see $\S 5.3$ ), but when $\epsilon$ is not small it is possible to find this function $f_{Q}$ by numerical approximations only.

In order to extend the definition of $f_{Q}$ to the whole stability zone of the periods $P$ and $Q * P$, one may write

$$
a^{\prime}=f_{Q}(a)
$$

whenever $a$ and $a^{\prime}$ belong to the stability zone of $P$ and $Q * P$ respectively, and $s_{Q * P}\left(a^{\prime}\right)=s_{P}(a)$, the parameter of stability $s$ being defined as in equation (2.4). The previous definition (3.11) corresponds to $s_{P}(a)=s_{Q * P}\left(a^{\prime}\right)=0$.

This pointwise construction of $f_{Q}$ is possible owing to the property of internal similarity and to the fact that the zones of stability of the various periods do not overlap. It is apparently true from numerical calculations that these stability zones are dense. Thus one may assume (and we shall do it) that $f_{Q}$ may be defined by continuity for any value of $a$. (As we already pointed out in $\S 2$, a similar construction for mapping $* P$ is not possible; we may write $a_{Q * P}=\vec{f}_{P}\left(a_{Q}\right)$ instead of (3.11), but we cannot extend it to the whole stability zone.)

If $q$ is the period of the sequence $Q$, obviously $k\left(a^{\prime}\right)=q k(a)$. The solution of

$$
a_{*}=f_{Q}\left(a_{*}\right)
$$

corresponds to a sequence $P$ such that $Q * P=P$; whence $P=Q^{* \infty}$, where the symbol $Q^{* \infty}$ represents the infinite sequence obtained as the limit of the sequences $Q^{* N}$ when $N$ goes to infinity ${ }^{\dagger}$. If $a_{n}=a_{\mathrm{Q}^{* n}}$, then

$$
a_{n+1}=f_{Q}\left(a_{n}\right) \simeq a_{*}-\left(a_{*}-a_{n}\right) \mathrm{d} f /\left.\mathrm{d} a\right|_{a=a_{*}}
$$

when $n$ is large. This yields at once the bifurcation velocity

$$
\delta_{Q}=\left(\mathrm{d} f_{Q} /\left.\mathrm{d} a\right|_{a=a_{*}}\right)^{-1} .
$$

More generally, if we consider sequences $P_{1} * Q^{* n} * P_{2}$ corresponding to parameters $b_{n}=a_{P_{1} * Q^{* n} * P_{2}}$, we have $b_{n+1}=\left(f_{P_{1}} \circ f_{Q} \circ f_{P_{1}}^{-1}\right)\left(b_{n}\right)$ as $b_{n}=f_{P_{1}} \circ f_{Q}^{n} \circ f_{P_{2}}\left(a_{\text {blank }}\right)$. The symbol $\circ$ denotes the composition law for applications: $(f \circ g)(x)=f(g(x))$. The fixed point is $b_{*} \neq a_{*}$ corresponding to the infinite sequence $P_{1} * Q^{* \infty}$, but because of the chain rule the bifurcation velocity is again $\delta_{Q}$. As already pointed out, $P_{2}$ plays no role.

Notice that $\delta_{Q}$ and $\nu_{Q}$ are not only functions of period $q$ but of the sequence $Q$ itself. Two sequences of the same period $q$ have distinct bifurcation velocities. For example $\delta_{R L^{2}} \neq \delta_{R L R}$. This will be shown for small $\epsilon$ in $\S 5$.

We are now able to derive the commutation law

$$
\delta_{Q_{1} * Q_{2}}=\delta_{Q_{2} * Q_{1}}
$$

$\doteqdot$ We shall not enter here into the rigorous definition of infinite sequences. This has already been done in $I$. 
and the corresponding law for critical exponents

$$
\nu_{Q_{1} * Q_{2}}=\nu_{Q_{2} * O_{1}}
$$

We have

$$
f_{Q_{1} * Q_{2}}=f_{Q_{1}} \circ f_{Q_{2}}, \quad f_{Q_{2} * O_{1}}=f_{Q_{2}} \circ f_{Q_{1}}
$$

where $\circ$ denotes again the usual composition law.

Let $a_{*}$ and $b_{*}$ be the fixed points for $f_{Q_{1} * Q_{2}}$ and $f_{Q_{2} * Q_{1}}$ respectively. Obviously

$$
b_{*}=f_{Q_{2}}\left(a_{*}\right), \quad a_{*}=f_{Q_{1}}\left(b_{*}\right) .
$$

As

$$
\begin{aligned}
\left(\delta_{Q_{1} * Q_{2}}\right)^{-1} & =\mathrm{d} f_{Q_{1} * Q_{2}} /\left.\mathrm{d} a\right|_{a=a_{*}}=\mathrm{d} f_{Q_{1}} /\left.\mathrm{d} a\right|_{a=f Q_{2}\left(a_{*}\right)} \times \mathrm{d} f_{Q_{2}} /\left.\mathrm{d} a\right|_{a=a_{*}} \\
& =\mathrm{d} f_{Q_{1}} /\left.\mathrm{d} a\right|_{a=b_{*}} \times \mathrm{d} f_{Q_{2}} /\left.\mathrm{d} a\right|_{a=a_{*}}
\end{aligned}
$$

is now a symmetric function of $a_{*}$ and $b_{*}$, we easily obtain results (3.15) and (3.16).

The fixed points $a_{*}$ and the universal metric constants $\nu_{Q}$ and $\delta_{Q}$ are known once the function $f_{Q}$ is known. Actually, even in the simplest cases it is not possible to get this function $f_{Q}$ exactly. The first-order correction when $\epsilon$ is small is given in $\S 5$. When $\epsilon$ is not small, we suggest different kinds of approximation schemes which are based on conjugation law (3.4) and are to be compared with the usual renormalisation methods. This will be done in $\S 4$.

\subsection{Analogies and differences with critical phenomena}

We end this section with some remarks. Parameter $a$ plays in bifurcation problems the role of temperature-or coupling constants-in the theory of phase transitions, and operation $f_{Q}$ is analogous to the recurrence relation which renormalises coupling constants. Similarly the critical temperature corresponds to the fixed point of $f_{Q}$, and exponent $\nu_{Q}$ is related to the linearised expression for $f_{Q}$ near $a_{*}$.

Nevertheless, there is a big difference, as there are an infinite number of operations $f_{Q}$. Two functions $f_{Q_{1}}$ and $f_{Q_{2}}$ define two renormalisations which have different critical points and critical exponents.

If $a_{Q_{1}^{* \infty}}$ and $a_{Q_{2}^{* \infty}}$ are the fixed points of $f_{Q_{1}}$ and $f_{Q_{2}}$, then function $k(a)$ diverges near $a_{Q_{1}^{* \infty}}$ and $a_{Q_{2}^{* \infty}}$, then near $f_{Q_{2}}\left(a_{Q_{1}^{* \infty}}\right), f_{Q_{2}^{* 2}}\left(a_{Q_{1}^{* \infty}}\right), \ldots, f_{Q_{1}}\left(a_{Q_{2}^{* \infty}}\right), \ldots$ and more generally for an infinite number of values of the parameter. The set of points for which $k(a)$ is infinite is stable by all operations $f_{Q}$ and has the power of the continuum (even if these points are nowhere dense; see I).

In the theory of phase transitions, the renormalisation is a transformation of the coupling constants of some functional (in our case the coefficients of the Taylor expansion of the transform near its maximum could be seen as more or less similar to these coupling constants). This transformation can be made infinitesimal, so that it defines a semi-flow in the space of the coupling constants, or it can be made by finite steps in the so-called 'real space' renormalisation. In the problem we consider here, the discreteness is fundamental- $f_{R}$ multiplies any period by $2, f_{R L}$ by 3 , and so on-and the behaviour of function $k(a)$ (length of the period as a function of $a$ ) near a critical point (that is near $a=a_{*}$ ) is not

$$
k(a) \sim\left|a-a_{Q^{* \infty}}\right|^{\nu_{O}}
$$


but rather of the form $(3.10)$

$$
k(a) \sim\left|a-a_{Q^{* \infty}}\right|^{\nu} \operatorname{og}_{Q}\left(\ln \left|a_{Q^{* \infty}}-a\right| / \ln \delta_{Q}\right)
$$

where $g_{Q}$ is periodic $\left(g_{Q}(x+1)=g_{Q}(x)\right)$ and has a very complicated structure. (For a discussion of phase transitions, see, for example, Domb and Green (1976)).

\section{Approximate calculations of the function $f_{Q}$}

In this section we investigate various approximations for computing the function $f_{Q}$ that allow one to derive the quantities $\delta_{Q}$ and $\nu_{Q}$ (equations (3.9) and (3.13), (3.14)). Using rather simple arguments, we may approximate the raw numerical results with a relative error that may be of the order of one or two per cent. All our approximation schemes may be refined indefinitely, at least in principle. The numerical evidence shows that the next-order calculations yield improved numerical values, so that we may believe that these calculations do really converge towards well defined results which are actually independent of the approximation scheme.

We describe first our calculations of $a_{Q^{* \infty}}$ and $\delta_{Q}$ for $Q=R$ and the $C^{\infty}$ endomorphism $\mathscr{T}_{a}(x)$ with a single maximum

$$
\mathscr{T}_{a}(x)=1-a x^{2} .
$$

We then explain how these calculations must be modified in the case $Q=R L$. Although the extension of these computations to other sequences does not involve any difficulty, at least in principle, we shall not do it, as we have no numerical results for comparison.

At the end of the section, we consider the nearly broken linear case $(\epsilon \sim 0)$ defined in equation (1.1); all the methods we propose give the same result at the first order in $\epsilon$, and we shall see in $\S 5$ that it is the first term of a systematic expansion near $\epsilon=0$. We shall not restrict this calculation to the sequences $Q=R$ and $Q=R L$ as its extension to any $Q$ is straightforward.

The approximation schemes we developed for $\epsilon=1$ may be also used for $\epsilon \neq 1$ or $\epsilon$ not too small. However, when this exponent is no longer an integer, many expressions become very complicated and are difficult to handle from a practical point of view, but the principle of the method remains unchanged. We expect a good convergence to the exact value for small $\epsilon$. The inaccuracy worsens as $\epsilon$ increases.

Finally, we give the exact expression for functions $f_{Q}$ for the piecewise linear mappings with a flat top. Their derivation will be done in a forthcoming publication.

\subsection{Quadratic case and $Q=R$}

Let us consider the endomorphism $\mathscr{T}_{a}(x)=1-a x^{2}$ in the interval $[1,2]$ of values of the parameter $a$. If $x$ belongs to the interval $I(a) \equiv[1-a, 1]$, then $\mathscr{T}_{a}(x) \in I(a)$ so that the interval $I(a)$ is stable under the action of $\mathscr{T}_{a}$, and $\mathscr{T}_{a}$ has a single extremum in this stable interval.

Let us now consider the range of values of the parameter defined by $1<a<a_{0}$, where $a_{0}$ is the real root of the polynomial $X^{3}-2 X^{2}+2 X-2$. In this range of values of $a$, two disconnected intervals exist, $I_{1}(a) \equiv[1-a, a-1]$ and $I_{2}(a) \equiv$ $\left[1-a+2 a^{2}-a^{3}, 1\right]$, which are both stable under the action of $\mathscr{T}_{a}^{2}$, and $\mathscr{T}_{a}^{2}$ has a single extremum in each of these intervals; of course $\mathscr{T}_{a}\left(I_{1}(a)\right) \subset I_{2}(a)$ and $\mathscr{T}_{a}\left(I_{2}(a)\right) \subset I_{1}(a)$. This helps one to understand the way in which the law of internal similarity holds: for a 
given value of $a$, there exists a value $A$ of the parameter corresponding to a mapping $\mathscr{T}_{A}$ and a homeomorphism $h_{1}$ between $I_{1}(a)$ and $I(A)$ such that

$$
\mathscr{T}_{a}^{2}(x)=h_{1} \circ \mathscr{T}_{A}(x) \circ h_{1}^{-1}
$$

(see also figure 5).
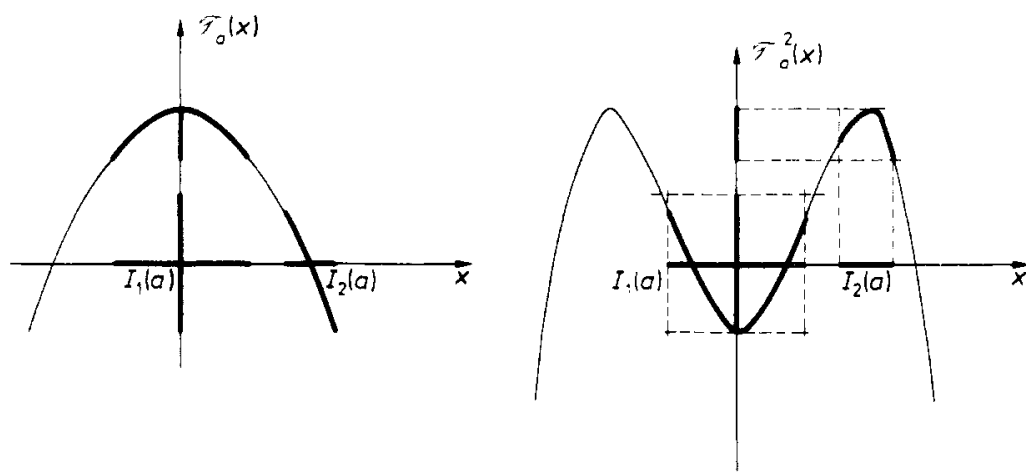

Figure 5. Segments $I_{1}(a)$ and $I_{2}(a)$ stable by transformation $\mathscr{T}_{a}^{2}$ for a given $a$. They are represented as full lines with the corresponding parts of $\mathscr{T}_{a}(x)$ and $\mathscr{T}_{a}^{2}(x)$.

To our knowledge, there is no way of proving the existence of such a pointwise homeomorphism. This proof would require, in particular, a knowledge of all the invariant sets of $\mathscr{T}_{a}^{2}$ and $\mathscr{T}_{A}$ in $I_{1}(a)$ and $I(\boldsymbol{A})$. In what follows we shall adopt a 'practical' point of view and try to find in an approximate way the homeomorphism $h_{1}$ and the function $f_{R}(a)$ that gives the value of $A$ by imposing a limited number of conditions to $h_{1}$ and $f_{R}(A)$. As we shall choose very simple functions for $h_{1}$, we shall assume implicitly that, at least for some values of $a, h_{1}$ is not a 'wild' function and can be actually fairly well approximated by this simple function.

\section{Remarks}

1. The same holds for the inverval $I_{2}(a)$. We look for a homeomorphism $h_{2}$ of $I(A)$ onto $I_{2}(a)$ such that

$$
h_{2}(I(A))=I_{2}(a), \quad h_{2}^{-1} \circ \mathscr{T}_{a}^{2} \circ h_{2}=\mathscr{T}_{A} .
$$

2. We are not able to say whether conjugation implies for $h_{1}$ and $h_{2}$ more than simple continuity. We think that, actually, $h_{1}$ and $h_{2}$ need to be differentiable at least.

3. To find the function $f_{R}$ is equivalent to looking for homeomorphisms $h_{1}$ and $h_{2}$ (equations (4.2) and (4.3)). Practically, there is no hope of getting them from a series expansion. Even for the reduced functions $\mathscr{T}_{a}(x)=1-a x^{2}$ the homeomorphisms cannot be polynomials or rational functions, as can be seen by simple consideration of the degrees and factorisations for the polynomials that conjugation (4.2) or (4.3) would require.

We are led to do simple approximations. In the following, we indicate several possibilities.

4.1.1. Centred renormalisation with one parameter. We assume that conjugation law (4.2) holds and that in a first approximation $h_{1}$ is linear. Keeping in $\mathscr{T}_{a}^{2}(x)$ terms up to 
second order, as (4.2) must hold for small $x$, we get

$$
1-a+2 a^{2} h_{1}^{2}(x)=h_{1}\left(1-A x^{2}\right)
$$

the solution of which is $h_{1}: x \rightarrow(1-a) x$.

Parameters $A$ and $a$ are related through

$$
A=2 a^{2}(a-1)
$$

which yields an implicit and approximate expression for $f_{R}$. From (3.13) and (3.14)

$$
\left.a_{*}=(1+\sqrt{3}) / 2 \quad \text { (fixed point } a_{*}=2 a_{*}^{2}\left(a_{*}-1\right)\right)
$$

and

$$
\delta_{R}^{-1}=\partial A /\left.\partial a\right|_{a=a_{*}}=(4-\sqrt{3}) / 13 .
$$

We get $a_{*}$ and $\delta_{R}^{-1}$ with an error of $2 \cdot 5 \%$ and $19 \%$ respectively. The results are shown

\begin{tabular}{|c|c|c|c|c|c|c|}
\hline Method of calculation & $\delta_{R}^{-1}$ & $\begin{array}{l}\text { Relative } \\
\text { error } \\
\text { in } \delta_{R}^{-1}(\%)\end{array}$ & $\begin{array}{l}\text { Critical } \\
\text { point } \\
a_{R^{* \infty}}\end{array}$ & $\begin{array}{l}\text { Relative } \\
\text { error } \\
\text { in } a_{R^{* \infty}}(\%)\end{array}$ & $\gamma_{R}^{-1}$ & $\begin{array}{l}\text { Relative } \\
\text { error } \\
\text { in } \gamma_{R}(\%)\end{array}$ \\
\hline \multicolumn{7}{|l|}{$\begin{array}{l}\text { Centred } \\
\text { renormalisation }\end{array}$} \\
\hline 1 parameter & $0 \cdot 1745$ & 19 & 1.366025 & $2 \cdot 5$ & 0.366025 & 8 \\
\hline 2 parameters & 0.2064 & 4 & 1.400030 & 0.08 & 0.394628 & $1 \cdot 2$ \\
\hline 3 parameters & $0 \cdot 2214$ & 3 & 1.401393 & 0.02 & 0.403403 & 1 \\
\hline \multicolumn{7}{|l|}{$\begin{array}{l}\text { Non-centred } \\
\text { renormalisation }\end{array}$} \\
\hline 1 parameter & $0 \cdot 2265$ & 6 & 1.457107 & 4 & & \\
\hline 2 parameters & $0 \cdot 2117$ & $1 \cdot 5$ & $1 \cdot 402414$ & 0.09 & & \\
\hline 3 parameters & 0.2133 & 0.4 & 1.401003 & 0.01 & & \\
\hline \multicolumn{7}{|l|}{$\begin{array}{l}\text { Non-linear } \\
\text { homeomorphism }\end{array}$} \\
\hline 1 parameters & $0 \cdot 1874$ & 12 & 1.384574 & 1 & & \\
\hline \multicolumn{7}{|l|}{$\begin{array}{l}\text { 1st equality of } \\
\text { slopes }\end{array}$} \\
\hline 1 parameters & $0 \cdot 1952$ & 9 & $1 \cdot 390388$ & $0 \cdot 7$ & & \\
\hline \multicolumn{7}{|l|}{$\begin{array}{l}\text { 2nd equality of } \\
\text { slopes }\end{array}$} \\
\hline 1 parameter & $0 \cdot 2167$ & 1 & 1.401420 & 0.02 & & \\
\hline $\begin{array}{l}\text { Experimental } \\
\text { (measured) value }\end{array}$ & \multicolumn{2}{|l|}{0.21416937} & \multicolumn{2}{|c|}{1.40115518909} & \multicolumn{2}{|l|}{0.399535} \\
\hline
\end{tabular}
in table 3 , row 1 .

Table 3. Comparison of the different methods of renormalisation for the set of sequences $R^{* n}$.

4.1.2. Non-centred renormalisation with one parameter. We proceed in the same way as above, except that we have (4.3) as a conjugation law. In the interval $I_{2}(a), \mathscr{T}_{a}^{2}$ reaches its maximum for the positive solution $x_{0}$ of $\mathscr{T}_{a}\left(x_{0}\right)=0$. Homeomorphism $h_{2}$ is assumed 
to be linear:

$$
h_{2}(x)=x_{0}+\lambda x .
$$

The choice of the constant term in this expression ensures that the extremum of $\mathscr{T}_{a}^{2}$ is mapped on the critical point of $\mathscr{T}_{A}$. For the reduced mapping (4.1) we get $x_{0}=a^{-1 / 2}$, $x_{0}+\lambda=1$ (this last condition ensures that the common right boundaries of $I_{2}(a)$ and $I(A)$ are mapped onto each other).

Then, by imposing again that the quadratic terms in $\mathscr{T}_{a}^{2}$ and $h_{2} \circ \mathscr{T}_{A} \circ h_{2}^{-1}$ are the same, one obtains

$$
A=4 a^{2}\left(1-a^{-1 / 2}\right) .
$$

This yields, by using (3.13) and (3.14), the following approximate expressions for $a_{*}$ and $\delta_{R}^{-1}: a_{*}=(3+2 \sqrt{2})$ (relative error $4 \%$ ), $\delta_{R}^{-1}=(3-\sqrt{2}) / 7$ (relative error $6 \%$ ) (see table 3 , row 4 ).

4.1.3. Centred renormalisation with serveral parameters. To get a better approximation, we may consider a larger set of transformations depending on several parameters $a, b$, $c, \ldots$ :

$$
T_{a, b, \ldots \ldots}(x)=1-a x^{2}+b x^{4}+c x^{6}+\ldots
$$

The coefficients of $T_{a, b, c, \ldots}^{2}(x)$ are polynomials in $a, b, c, \ldots$, and the stable segments $I$, $I_{1}, I_{2}$ depend now on $a, b, c, \ldots$.

If we look for a centred approximation and assume that $h_{1}$ is linear

$$
T_{a, b, c \ldots}^{2}\left(h_{1}(x)\right)=h_{1}\left(T_{A, B, C, \ldots}(x)\right), \quad x \in I(a, b, c, \ldots)
$$

we get

$$
h_{1}(x)=u_{0} x, \quad A=u_{2} u_{0}, \quad B=u_{4} u_{0}^{3}, \quad C=u_{6} u_{0}^{5}
$$

where

$$
T_{a, b, c, \ldots}^{2}(x)=u_{0}-u_{2} x^{2}+u_{4} x^{4}+u_{6} x^{6}+\ldots
$$

and the $u_{i}$ 's are polynomials in $a, b, c, \ldots$

Then the fixed point is the solution of $A=a, B=b, C=c, \ldots$, and the unstable eigenvalues of the matrix of the derivatives give $\delta_{R}$.

For example, with two parameters $a, b$ we get

$$
u_{0}=1-a+b, \quad u_{2}=4 a b-2 a^{2}, \quad u_{4}=-a^{3}+6 a^{2} b-2 a b+4 b^{2} .
$$

Then

$$
A=(1-a+b)\left(4 a b-2 a^{2}\right), \quad B=(1-a+b)^{3}\left(-a^{3}+6 a^{2} b-2 a b+4 b^{2}\right) \text {. }
$$

The calculations give very good results (see table 3 , row 2 ). The accuracy is improved further with three parameters (table 1, row 3), but approximations, though more accurate, become very complicated with more parameters.

We have plotted on figure 6 the critical line and fixed point in the plane $(a, b)$. The value $a_{*}$ for $\mathscr{T}_{a}(x)=1-a x^{2}$ corresponds to $b=0$ on the critical line. The critical line stops at another fixed point $a=0, b=-1.545$ corresponding to the sequence $R^{* \infty}$ and the transformation $T_{b}(x)=1+b x^{4}$. 


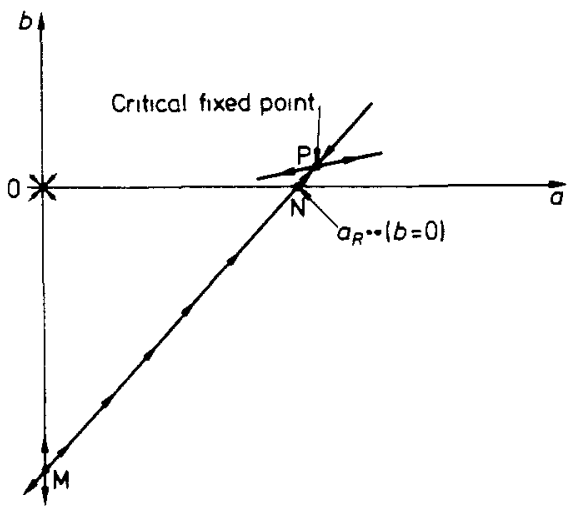

Figure 6. Two-parameter centred renormalisation. The recurrence equations (4.7) have interesting fixed points. The origin is attractive; the fixed point of $T_{b}=1+b x^{4}$ is repulsive. The critical fixed point $P$ is unstable except for the critical line which goes through the fixed points of the one-parameter transformation $T_{b}=1+b x^{4}$ (point $\mathrm{M}$ ) and $\mathscr{T}_{a}=1-a x^{2}$ (point N).

\section{Important remarks}

1. This procedure is similar to that used in phase transition theory, where one assumes that the renormalised Hamiltonian is accurately described by a small number of 'relevant' coupling constants. As we pointed out in the introduction, if we were looking to $a_{R^{* \infty}}$ and $\delta_{R}$ for another $C^{\infty}$ transformation, for example

$$
T_{a}(x)=1-a \sinh ^{2} x \sim 1-a\left(x^{2}+\frac{1}{3} x^{4}\right)
$$

we should recover the same $\delta_{R}$. The fixed point $a_{R^{* \infty}}$ is approximately at the intersection of the critical line with $b=-a / 3$.

2. Non-centred renormalisation with several parameters. The same method holds for $I_{2}$ and $h_{2}$, but, as $T_{a, b, c, \ldots}^{2}$ is no longer symmetric around $x_{0}$, we must include odd powers of $x$. For example, with three parameters $a, b, c$

$$
T_{a, b, c}(x)=1-a x^{2}+b x^{3}+c x^{4}
$$

and $x_{0}(\neq 0)$ is the solution of $T_{a, b, c}\left(x_{0}\right)=0$. Expanding $T_{a, b, c}^{2}$ around $x_{0}$ we get

$$
T_{a, b, c}^{2}\left(x_{0}+y\right)=1-v_{2} y^{2}+v_{3} y^{3}+v_{4} y^{4}+\ldots
$$

where again $v_{2}, v_{3}, v_{4}$ are functions of $a, b, c$ and

$$
A=v_{2}\left(1-x_{0}\right), \quad B=v_{3}\left(1-x_{0}\right)^{2}, \quad C=v_{4}\left(1-x_{0}\right)^{3} .
$$

We did calculations with two and three parameters (table 3, rows 5 and 6). The latter gives $\delta_{R}^{-1}$ with an error of $0.4 \%$ and $a_{R^{* \infty}}$ with an error of $0.01 \%$. This method is the most accurate among all we tried, and results should be still better with more parameters. Unfortunately, this method yields the longest renormalisation formulae!

4.1.4. Nonlinear homeomorphism. Up to now, we have approximated homeomorphisms $h_{1}$ and $h_{2}$ by linear functions. This is reasonable, as this is true asymptotically. Nevertheless we may ask whether a more elaborate choice for $h_{1}$ and $h_{2}$ gives better accuracy. As an example, we look here at $a_{*}$ and $\delta_{R}^{-1}$ when the renormalisation is 
centred and with the homeomorphism $h_{1}^{-1}(x)=\lambda x+\mu x^{3}$. From $h_{1}^{-1}\left(T_{a}^{2}(x)\right)=$ $T_{A}\left(h_{1}^{-1}(x)\right)$ and neglecting all terms $x^{n}, n \geqslant 5$ we get the equation for $A$

$$
\alpha(\dot{a}) A^{2}+\beta(a) A+\gamma(a)=0
$$

where

$$
\begin{aligned}
& \alpha(a)=8 \\
& \beta(a)=16 a^{2}-32 a^{3}+83 a^{4}-151 a^{5}+222 a^{6}-318 a^{7}+255 a^{8}-75 a^{9} \\
& \gamma(a)=64 a^{6}-208 a^{7}+368 a^{8}-608 a^{9}+736 a^{10}-464 a^{11}+112 a^{12}
\end{aligned}
$$

whence $a_{*}$ and $\delta_{R}^{-1}$ (table 3 , row 7). The accuracy is better than with centred (linear) renormalisation with one parameter, but not as good as the other approximations we described in $\S \S 4.1 .2$ and 4.1.3.

4.1.5. Equality of the slopes. We deal only with sequences $R^{* n}$. The parameter $s_{2^{n}}$ (equations (2.3) and (2.4)) which defines the stability zone for $R^{* n}$ is the slope of $\mathscr{T}_{a}^{2 n}(x)$ at one point of the period.

Sequence $b$ (equal to the fixed point) is stable if $\left|s_{1}\right|<1$, i.e. $-\frac{1}{4}<a<\frac{3}{4}$, where $s_{1}(a)=1-(1+4 a)^{1 / 2}$.

Sequence $R$ is stable if $\left|s_{2}\right|<1$, i.e. $\frac{3}{4}<a<\frac{5}{4}$, where $s_{2}(a)=4(1-a)$, and $s_{4}(a)$ is given by the implicit expression

$$
1+2 a^{2}-3 a^{3}+3 a^{4}+a^{6}+\frac{1}{16} s_{4}(a)\left(a^{4}-a^{3}-a^{2}-3\right)+\frac{s_{4}^{2}(a)}{16^{2}}\left(3-a^{2}\right)-\frac{s_{4}^{3}(a)}{16^{3}}=0
$$

which holds for $\frac{5}{4}<a<\bar{a}$ (destabilisation value).

When $-\frac{1}{4}<A<\frac{3}{4}$, the function $f_{R}$ is given implicitly by $s_{1}(A)=s_{2}(a)$, i.e.

$$
a=\left[3+(1+4 A)^{1 / 2}\right] / 4
$$

and when $\frac{3}{4}<A<\frac{5}{4}, s_{2}(A)=s_{4}(a)$ gives for $a=f_{R}(A)$ the implicit equation

$$
\begin{aligned}
A^{3}+A^{2}\left(9-4 a^{2}\right) & +A\left(27+24 a^{2}+16 a^{3}-16 a^{4}\right)+27 \\
+ & 108 a^{2}-208 a^{3}+208 a^{4}-192 a^{5}+64 a^{6}=0 .
\end{aligned}
$$

The analytic form for $f_{R}(A)$ changes at $A=\frac{3}{4}$, though both $\mathrm{f}_{\mathrm{R}}$ and $d f_{R} / \mathrm{d} A$ are continuous. Using (4.9a) as an approximation for $f_{R}$, we get $a_{*}=(7+\sqrt{17}) / 8$ and $\delta_{R}^{-1}=(\sqrt{17}-1) / 16$. When $(4.9 b)$ is used, the accuracy is much better (actually, it is one of the best approximations; see table 3, rows 8-9), as the expression $(4.9 b)$ for $f_{R}$ corresponds to an interval which is nearer the limit point $a_{R^{* \infty}}$.

\section{Remark}

One may notice that even the simplest methods sketched in this subsection give a good order of magnitude for $a_{R^{* \infty}}$ and $\delta_{R}$; more elaborate methods (mainly non-centred linear renormalisation with three parameters, and the slope method of equation $(4.9 b)$ ) give very accurate results. Nevertheless we are not able a priori to tell which method will give the best result. This is mainly due to our lack of knowledge of the manner in which these approximations converge to the exact result.

One could improve these approximation schemes by increasing the number of parameters, but again we do not know if this will actually increase the precision. We have no knowledge of a proof of the convergence of all these methods to a single result. 


\subsection{Quadratic case $(\epsilon=1$ and $Q=R L)$}

We indicate briefly how the above prescriptions are modified when we replace $R$ by $R L$.

The main point is that there are three disconnected intervals $J_{1}, J_{2}, J_{3}$ which are stable by $\mathscr{T}_{a}^{3}(x), \mathscr{T}_{a}\left(J_{1}\right) \subset J_{2}, \mathscr{T}_{a}\left(J_{2}\right) \subset J_{3}, \mathscr{T}_{a}\left(J_{3}\right) \subset J_{1}$ for a range of the parameter $a_{1}<a<a_{1}^{\prime} ; a_{1}$ and $a_{1}^{\prime}$ being the values of the parameter $a$ corresponding to sequences $R L$ and $R L * R L^{\infty}$ respectively. On each interval $\mathscr{T}_{a}^{3}$ has one extremum only and only one of these (say $J_{1}$ ) is centred at $x=0$. Choosing any one of these intervals $J_{i}$ ( $i=1,2,3$ ), we may write $\mathscr{T}_{a}^{3} \sim \mathscr{T}_{A}$, which implies the existence of a homeomorphism $j_{i}$ between $I(A)$ and $J_{i}(a)$ such as

$$
j_{i}(I(A))=J_{i}(a), \quad \mathscr{T}_{a}^{3}\left(j_{i}(x)\right)=j_{i}\left(\mathscr{T}_{A}(x)\right), \quad \forall x \in I(A) .
$$

We list here the analytic expressions for the simplest approximations for $f_{R L}$ :

$A=4 a^{3}(a-1)\left(a^{3}-2 a^{2}+a-1\right) \quad$ centred renormalisation with one parameter

$A=8 a^{3}\left(1-a+\frac{1}{\sqrt{a}}\right)$ first non-centred renormalisation with one parameter

$A=16 a^{3}\left(1+\frac{1}{\sqrt{a}}\right)\left[1-\frac{1}{\sqrt{a}}\left(1+\frac{1}{\sqrt{a}}\right)^{1 / 2}\right] \begin{aligned} & \text { second non-centred renormalisation with one } \\ & \text { parameter }\end{aligned}$ $A=\frac{\left[4 a-7+4 a(4 a-7)^{1 / 2}\right]^{2}-1}{4}$ equality of slopes $\left(s_{1}(A)=s_{3}(a)\right)$.

Again $a_{R L^{* \infty}}$ is known with a good accuracy and we have a good order of magnitude for $\delta_{R L}^{-1}$. We get better results with a centred renormalisation with two parameters (table 4 ).

Table 4. Comparison of the different methods of renormalisation for the set of sequences $(R L)^{* n}$.

\begin{tabular}{|c|c|c|c|c|c|c|}
\hline Method of calculation & $\delta_{R L}^{-1}$ & $\begin{array}{l}\text { Relative } \\
\text { error } \\
\text { in } \delta_{R L}^{-1}(\%)\end{array}$ & $\begin{array}{l}\text { Critical } \\
\text { point } \\
a_{R L^{* *}}\end{array}$ & $\begin{array}{l}\text { Relative } \\
\text { error } \\
\text { in } a_{R L^{* \infty}}(\%)\end{array}$ & $\gamma_{R L}^{-1}$ & $\begin{array}{l}\text { Relative } \\
\text { error } \\
\text { in } \gamma_{R L}(\%)\end{array}$ \\
\hline \multicolumn{7}{|l|}{$\begin{array}{l}\text { Centred } \\
\text { renormalisation }\end{array}$} \\
\hline 1 parameter & 0.015077 & 17 & $1 \cdot 784998$ & 0.08 & 0.099953 & 7 \\
\hline 2 parameters & 0.018118 & $0 \cdot 1$ & $1 \cdot 786466$ & $0 \cdot 0015$ & 0.107834 & 0.04 \\
\hline \multicolumn{7}{|l|}{$\begin{array}{l}\text { 1st non-centred } \\
\text { renormalisation }\end{array}$} \\
\hline 1 parameter & $0 \cdot 017176$ & 5 & 1.787164 & 0.04 & & \\
\hline \multicolumn{7}{|l|}{$\begin{array}{l}\text { 2nd non-centred } \\
\text { renormalisation }\end{array}$} \\
\hline 1 parameter & 0.017721 & 2 & 1.787696 & 0.07 & & \\
\hline \multicolumn{7}{|l|}{ Equality of slopes } \\
\hline 1 parameter & 0.016229 & 10 & 1.785972 & 0.03 & & \\
\hline $\begin{array}{l}\text { Experimental } \\
\text { (measured) value }\end{array}$ & 0.0181005 & & 1.786440255 & & $0 \cdot 107789$ & \\
\hline
\end{tabular}


These methods might be extended to all sequences $Q$ of period $q$. In particular, it has already been shown that in some range of values of the parameter $a, q$ disconnected intervals exist; each of them is stable under the action of $T_{a}^{(a)}(x)$, and this function has a single critical point in each interval (Guckenheimer 1977).

\subsection{Study of small $\epsilon$}

The same methods are developed for the mapping $\mathscr{T}_{a}(x)=1-a|x|^{1+\epsilon}$ when $\epsilon$ is small.

By the centred and non-centred renormalisation with one parameter we get for $f_{R}$ $A=a^{2}(1+\epsilon)(a-1)^{\epsilon} \quad$ and $\quad A=a^{2}(1+\epsilon)^{1+\epsilon}\left(1-a^{-1 /(1+\epsilon)}\right)^{\epsilon}$

respectively, which gives to first order the same results:

$$
\begin{aligned}
& a_{R^{* \infty}}=1+\varphi, \quad \delta_{R}=2+\epsilon / \varphi \text { hence } \\
& \nu_{R}=-1+\epsilon / 2(\ln 2) \varphi
\end{aligned}
$$

where $\varphi$ is the solution of $\varphi+\epsilon \ln \varphi+\epsilon=0$. We shall see in $\S 5$ that this is the exact first-order term.

Similarly for $Q=R L$ we get

$$
\begin{aligned}
& A=a^{3}|a-1|^{\epsilon}(1+\epsilon)^{2}\left(a|a-1|^{1+\epsilon}-1\right)^{\epsilon} \text { and } \\
& A=a^{3}(1+\epsilon)^{2+\epsilon}\left|a-a^{-1 /(1+\epsilon)}-1\right|^{\epsilon}
\end{aligned}
$$

which gives for $a_{(R L)^{* \infty}}$ and $\delta_{R L}$ the same first-order terms

$$
a_{(R L) * \infty} \simeq a_{R L}+\varphi, \quad \delta_{R L} \simeq \epsilon_{a_{R L}} / \varphi
$$

where $a_{R L}$ is the root of $1-a_{R L}\left|a_{R L}-1\right|^{1+\epsilon}=0$, corresponding to the superstability point of sequence $R L$, and

$$
\varphi=\mathrm{e}^{-2}\left[\left(2 a_{R L}-1\right)\left(a_{R L}-1\right)\right]^{-1} a_{R L}^{-2 / \epsilon} .
$$

Again this is the precise first-order result as we shall see in $\S 5$. Notice that, in contrast to $\delta_{R}, \delta_{R L}$ increases infinitely for $\epsilon \rightarrow 0$; as a consequence the critical exponent $\nu_{R L}$ goes to zero.

As even the simplest methods seem to give very good results for small $\epsilon$, we may continue and do the same thing with any sequence $Q$ of period $q$. With the centred renormalisation method, as

$$
\begin{aligned}
\mathscr{T}_{a}^{(k)}(x)=\mathscr{T}_{a}^{k}(0) & +(-a)^{k}(1+\epsilon)^{k-1} \\
& \times \operatorname{sgn}\left(\mathscr{T}_{a}(0) \ldots \mathscr{T}_{a}^{(k-1)}(0)\right)\left|\mathscr{T}_{a}(0) \ldots \mathscr{T}_{a}^{(k-1)}(0)\right|^{\epsilon}|x|^{1+\epsilon}+\ldots
\end{aligned}
$$

we get

$$
\boldsymbol{A}=\left|\mathscr{T}_{a}(0) \ldots \mathscr{T}_{a}^{(k)}(0)\right|^{\epsilon} \operatorname{sgn}\left(\mathscr{T}_{a}(0) \ldots \mathscr{T}_{a}^{(k-1)}(0)\right)(1+\epsilon)^{k-1} a^{k}(-1)^{k-1}
$$

$\mathscr{T}_{a}(0), \ldots, \mathscr{T}_{a}^{(k-1)}(0)$ are all far from zero, but $\mathscr{T}_{a}^{(k)}(0)$ is small and can be written as

$$
\mathscr{T}_{a}^{(k)}(0)=\left(a-a_{Q}\right)(\mathrm{d} / \mathrm{d} a) \mathscr{T}_{a}^{(k)}(0)+\ldots
$$

where $a_{Q}$ is the solution of $\mathscr{T}_{a_{Q}}^{(k)}(0)=0$ and corresponds to the superstability point for sequence $Q$, whence the first-order approximation for $a_{*}$

$$
a_{*}-a_{Q} \sim \frac{\mathrm{e}^{-(k-1)} a_{*}^{-(k-1) / \epsilon}}{\left|\mathscr{T}_{a}(0) \ldots \mathscr{T}_{a}^{(k-1)}(0)\right|\left|(\mathrm{d} / \mathrm{d} a) \mathscr{T}_{a}^{k}(0)\right|}
$$


which generalises result (4.12) and is exactly expression (5.12). We recover that, when $Q \neq R, \delta_{Q} \rightarrow \infty$ (and $\nu_{Q} \rightarrow 0$ ) in the limit $\epsilon \rightarrow 0$.

\subsection{Piecewise linear mapping}

We consider the transformation $T_{r, \lambda}(x)$ depending on two parameters $r$ and $\lambda$ and defined by (figure 7)

$$
T_{r, \lambda}(x)= \begin{cases}\lambda x & x<1 \\ \lambda & 1<x<r \\ \lambda(1+r)-\lambda x & x<r .\end{cases}
$$

For $r=1$ we recover the so-called broken linear case $\epsilon=0$ that we studied in I. Perhaps the broken linear transform in the limit $r \neq 0, \lambda \rightarrow \infty$ could have something to do with the behaviour of $\mathscr{T}_{a}(x)$ when $\epsilon \rightarrow \infty$.

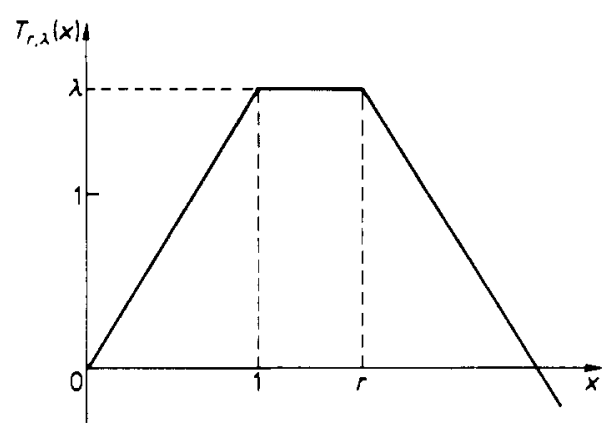

Figure 7. Plot of $T_{r, \lambda}(x)$ as a function of $x$.

All calculations may be carried out. The iterates of $T_{r, \lambda}$ are piecewise linear functions with flat extrema. With two parameters, the linear renormalisation is then exact. For $Q=R, T_{r, \lambda}^{2} \sim T_{R, \Lambda}$, where

$$
\Lambda=\lambda^{2}, \quad(R-1) /(R+1)=(\lambda+1)(r-1) /(\lambda-1)(r+1) .
$$

Similarly, fcr $Q=R L, T_{r, \lambda}^{3} \sim T_{R^{\prime}, \Lambda^{\prime}}$, with

$$
\Lambda^{\prime}=\lambda^{3}, \quad\left(R^{\prime}-1\right) /\left(R^{\prime}+1\right)=\left(\lambda^{2}+\lambda+1\right)(r-1) /\left(\lambda^{2}-\lambda-1\right)(r+1) .
$$

\section{Remark}

The fixed points of these recursion relations go to infinity, and it can be shown that in this case the length of the periods diverges logarithmically near the critical values of the parameter. More generally, for $Q$ with period $k$ and characteristic polynomial $P_{Q}(x)=$ $\alpha_{0} \ldots \alpha_{k-2} \mathscr{P}_{Q}(x)($ see $\& 2.2), T_{r, \lambda}^{k} \sim T_{R^{\prime \prime}, A^{\prime \prime}}$, with

$$
\Lambda^{\prime \prime}=\lambda^{k}, \quad\left(R^{\prime \prime}-1\right) /\left(R^{\prime \prime}+1\right)=\left(\lambda^{k}-1\right)(r-1) /(\lambda-1) P_{Q}(\lambda)(r+1) .
$$




\section{Study of the superstable periods for $\epsilon \sim 0$}

As we saw in the preceding sections, we need only the behaviour of $T_{a}$ near its maximum. Then, in $\S 4$, we considered the reduced transformation

$$
\mathscr{T}_{a}(x)=1-a|x|^{1+\epsilon}
$$

depending continuously on the parameter $\epsilon$. For $\epsilon=0$ we recover the piecewise linear mapping

$$
\mathscr{L}_{a}(x)=1-a|x|
$$

which we studied in some detail in I. The superstable (or MSS) parameters $a_{Q}$ are then the so-called $\lambda$ numbers. For small $\epsilon$ the parameter $a_{Q}$ for sequence $Q$ may be obtained by expansion near the $\lambda$ number of this period. The knowledge of $a_{Q}$ itself is not very important, but from equations (3.14) and (3.9) we may get the universal parameters $\delta_{O}$ and $\nu_{O}$ respectively. This is essentially what is explained in that section. The exponent $\epsilon$ is given and assumed to be small although $a$ is varying, and we shall call $a$ the parameter.

We use in a relaxed way the terminology first order, second order, . . . Rigorously it is not correct, as the dependence in $\epsilon$ will not be polynomial. When a quantity $\phi(\epsilon)$ may be written as a sum

$$
\phi(\epsilon)=\phi_{1}(\epsilon)+\phi_{2}(\epsilon)+\phi_{3}(\epsilon)+\ldots
$$

with

$$
\phi_{2}(\epsilon) / \phi_{1}(\epsilon) \rightarrow 0, \quad \phi_{3}(\epsilon) / \phi_{2}(\epsilon) \rightarrow 0, \ldots
$$

then $\phi_{1}(\epsilon), \phi_{2}(\epsilon), \phi_{3}(\epsilon)$ will be said to be of first, second and third-order respectively.

As the case $Q=R$ differs from the other sequences, it is studied separately in $\S 5.4$.

In the following, $\bar{a}_{Q}$ and $a_{Q}$ are the values of the superstable sequence when $\epsilon=0$ and $\epsilon \neq 0$ respectively; $x_{n}\left(\bar{a}_{Q}\right)=\mathscr{L}_{\bar{a}_{O}}^{(n)}(0)$ and $y_{n}\left(a_{Q}\right)=\mathscr{T}_{a_{O}}^{(n)}(0)$ are the iterates $(n=$ $1, \ldots, k)$ of the maximum when $\epsilon=0$ and $\epsilon \neq 0$ respectively.

\subsection{Shift of the parameters of superstable periods}

When $\epsilon$ is small, we rewrite $\left\{y_{n}\right\}$ and $a_{Q}$ as

$$
y_{n}=x_{n}+\epsilon z_{n}+\mathrm{O}\left(\epsilon^{2}\right), \quad a_{Q}=\bar{a}_{Q}+\epsilon \Delta a+\mathrm{O}\left(\epsilon^{2}\right)
$$

and to first order, the iteration formula (5.1) gives

$$
z_{n}=-\bar{a}_{Q}\left(\operatorname{sgn} x_{n-1}\right) z_{n-1}-\Delta a\left|x_{n-1}\right|-\bar{a}_{Q}\left|x_{n-1}\right| \ln \left|x_{n-1}\right|
$$

with 'sgn' being the sign function (equation (2.10)).

The solution of (5.4) with initial conditions $z_{0}=z_{1}=0$ is

$z_{n}=\sum_{i=2}^{n-1} \bar{a}_{Q}^{n-i} \alpha_{n-2} \ldots \alpha_{i-1} x_{i} \ln \left|x_{i}\right|+\left(\Delta a / \bar{a}_{Q}\right) \sum_{i=0}^{n-1} \bar{a}_{Q}^{n-1-i}(n-1-i) \alpha_{n-2} \ldots \alpha_{i}$

the first sum being zero when $n<3$.

As $Q$ is a $k$ period, $z_{k}=0$, whence the shift of the parameter is of order $\epsilon$ and

$$
\Delta a=-\frac{\sum_{i=2}^{k-1} \bar{a}_{Q}^{k-1} \alpha_{k-2} \ldots \alpha_{i-1} x_{i}\left(\bar{a}_{Q}\right) \ln \left|x_{i}\left(\bar{a}_{Q}\right)\right|}{\left(\mathrm{d} / \mathrm{d} \bar{a}_{Q}\right) x_{k}\left(\bar{a}_{Q}\right)}
$$


In (5.5) we have made explicit the dependence on the parameter $\bar{a}_{Q}$ and $x_{k}\left(\bar{a}_{Q}\right)=$ $\mathscr{P}_{Q}\left(\bar{a}_{Q}\right)=0$, where $\mathscr{P}(x)$ is defined in $(2.10)$.

Notice that, if $Q\left(=P_{1} * P_{2}\right)$ is non-primary, as $\mathscr{P}_{Q}\left(\bar{a}_{P_{1}}\right)=\mathscr{P}_{P_{1}}\left(\bar{a}_{P_{1}}\right) \mathscr{P}_{P_{2}}\left(\bar{a}_{P_{1}}^{k}\right)$, both numerator and denominator factorise in (5.5), $\bar{a}_{Q}=\bar{a}_{P_{1}}$ and $\Delta \bar{a}_{Q}=\Delta \bar{a}_{P_{1}}$. Again it is not possible to separate non-primary sequences by a first-order expansion. We shall see in $\S 5.3$ that the splitting $a_{P_{1} * P_{2}}-a_{P_{1}}$ is very small.

\subsection{Zone of stability}

Now we assume that $a_{Q}$ is known, $\left\{y_{n}\right\}$ is the sequence of the iterates of the maximum, and we look for a value $b$ of the parameter and for $k$ numbers $t_{0}(b), t_{1}(b), \ldots, t_{k-1}(b)$ $\left(t_{k}(b)=t_{0}(b)\right)$ such that

$$
\prod_{i=1}^{k}\left(\mathrm{~d} / \mathrm{d} t_{i}\right) \mathscr{T}_{b}\left(t_{i}\right)=\eta
$$

where $t_{i}=\mathscr{T}_{b}\left(t_{i-1}\right)$ is the $i$ th iterate of $t_{0}$, and $\eta= \pm 1$.

When $\eta=0, b=a_{O}$, although $b_{\mathrm{s}}$ and $b_{\mathrm{u}}$ mark the beginning $(\eta=+1)$ and end $(\eta=-1)$ of the stability zone of the period $Q$ in the parameter space. Equation (5.6) can be rewritten

$$
(-b)^{k}(1+\epsilon)^{k}\left|t_{1} \ldots t_{k}\right|^{\epsilon}\left(\operatorname{sgn} t_{1}\right) \ldots\left(\operatorname{sgn} t_{k}\right)=\eta .
$$

As $b \simeq a_{Q}$, then $t_{i} \simeq y_{i}$ and $\operatorname{sgn} t_{i}=\operatorname{sgn} y_{i}=\operatorname{sgn} x_{i}$ if $1 \leqslant i \leqslant k-1$. We have $t_{k}=t_{0} \approx 0$, and from (5.7)

$$
\left|t_{0}\right|=b^{-k / \epsilon}(1+\epsilon)^{-k / \epsilon} /\left|t_{1} \ldots t_{k-1}\right|
$$

with sgn $t_{0}=(-1)^{k} \eta \operatorname{sgn}\left(y_{1} \ldots y_{k-1}\right)$. Then the stability zone is very small, and its width is proportional to $b^{-k / \epsilon}$. Setting

$$
b-a_{Q}=a_{Q}^{-k / \epsilon} u \text {, and } t_{n}-y_{n}=a_{Q}^{-k / \epsilon} v_{n}
$$

the recursion formula (5.1) gives to first order

$$
v_{n}=-a_{Q} v_{n-1} \operatorname{sgn} x_{n-1}-u\left|x_{n-1}\right|
$$

with general solution (compare with (5.4))

$$
v_{n}=A \alpha_{n-2} \ldots \alpha_{0}\left(a_{Q}\right)^{n}+u \sum_{i=0}^{n-1}\left(a_{Q}\right)^{n-2-i}(n-1-i) \alpha_{n-2} \ldots \alpha_{i}
$$

$A$ being a parameter. With the initial conditions for $t_{0}$ above, we get

$$
v_{n}=u\left(\mathrm{~d} / \mathrm{d} a_{Q}\right) x_{n}\left(a_{Q}\right)+a_{Q}^{n-k} \mathrm{e}^{-k} \alpha_{n-2} \ldots \alpha_{0} /\left|y_{1} \ldots y_{k-1}\right| .
$$

The condition $v_{k}=v_{0}=t_{0} a_{Q}^{k / \epsilon}$ reads to first order $u\left(\mathrm{~d} / \mathrm{d} a_{Q}\right) x_{k}\left(a_{Q}\right)-\mathrm{e}^{-k} \alpha_{k-2} \ldots \alpha_{0} /\left|y_{1} \ldots y_{k-1}\right|=-\eta\left(\alpha_{k-2} \ldots \alpha_{0} \mathrm{e}^{-k}\right) /\left|y_{1} \ldots y_{k-1}\right|$.

(1) When $\eta=-1$ (end of stability zone) we get

$$
b_{\mathrm{u}}=a_{Q}+2\left(a_{Q}^{-k / \epsilon} \mathrm{e}^{-k} \alpha_{k-2} \ldots \alpha_{0}\right) /\left|y_{1} \ldots y_{k-1}\right|\left(\mathrm{d} / \mathrm{d} a_{Q}\right) x_{k}\left(a_{Q}\right)
$$

For example, if $k=3, Q=R L$ and

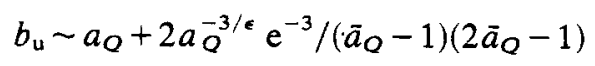


where $\bar{a}_{Q}=(1+\sqrt{5}) / 2$ is the golden number. Notice that, as $a_{Q}-\bar{a}_{Q} \sim \mathrm{O}(\epsilon), a_{Q}^{-k / \epsilon}$ and $\bar{a}_{Q}^{-k / \epsilon}$ are not equivalent in the limit $\epsilon \rightarrow 0$, although we may replace $y_{i}\left(a_{Q}\right)$ by $x_{i}\left(\bar{a}_{Q}\right)$. (2) When $\eta=+1$ (beginning of stability zone) we get $u=0$. The correction to the superstable parameter is at least of the order $\epsilon a_{Q}^{-k / \epsilon}$, and to a first approximation the beginning of the stability zone and the point of superstability coincide. To get the second-order term, we replace $(5.8)$ by

$$
b-a_{Q}=\epsilon v a_{Q}^{-k / \epsilon}, \quad t_{n}-y_{n}=a_{Q}^{-k / \epsilon}\left(v_{n}+\epsilon v_{n}^{\prime}\right)
$$

and keep in the iteration formula (5.1) all terms up to second order, getting

$$
b_{\mathrm{s}}=a_{Q}-\epsilon a_{O}^{-k / \epsilon} \mathrm{e}^{-\mathrm{k}} \alpha_{k-2} \ldots \alpha_{0} /\left|y_{1} \ldots y_{k-1}\right|\left(\mathrm{d} / \mathrm{d} a_{Q}\right) x_{k}\left(a_{Q}\right) .
$$

As $\alpha_{k-2} \ldots \alpha_{0}\left(\mathrm{~d} / \mathrm{d} a_{Q}\right) x_{k}\left(a_{Q}\right)>0$, we have $b_{\mathrm{s}}<a_{Q}<b_{\mathrm{u}}$. We thus verify that the width of the stability zone (that is $b_{\mathrm{s}}-b_{\mathrm{u}}$ ) is very small and reduces to a point when $\epsilon=0$. Moreover, when $\epsilon=0$ the measure of the total stability zone is zero as no period is stable. We expected this result as the set $\left\{\bar{a}_{Q}\right\}$ for $\epsilon=0$ is countable.

\subsection{Splitting of non-primary sequences}

Sequence $Q$ is now assumed to be primary with period $k>2, P$ is any other sequence, primary or not, of period $l$. We look for the value $a_{Q * P}$ of the parameter for which the period $Q * P$ is superstable. When $\epsilon=0, \overline{a_{Q * P}}=\bar{a}_{Q}$; near $\epsilon \sim 0$ ( $\$ \S 5.2$ and 4.3), $a_{Q * P}-a_{Q} \sim a_{Q}^{-k / \epsilon} a_{P}^{1 / \epsilon}$. This implies that $a_{Q * P_{1}}$ and $a_{Q * P_{1} * P_{2}}$ cannot be separated in a first-order approximation unless $a_{P_{2}}=1$ (which corresponds to $P_{2}=R$ ). This is of course no longer true when we take into account higher-order terms.

Proof. $\left\{t_{n}\right\}(n=1,2, \ldots, k l)$ is the sequence of iterates of the maximum for the value $a_{Q * P}$ of the parameter; we have $t_{k l}=0$ and $t_{k m} \neq 0$ (but small) if $1<m<l ;\left\{y_{n}\right\}$ denotes again the sequence of iterates of the maximum for $Q$ with parameter $a_{Q}$. We have $y_{k}=y_{2 k}=\ldots=y_{l k}=0$ and $y_{k m+r}=y_{r}(0 \leqslant r<k, 0 \leqslant m<l)$.

Let us write

$$
t_{n}=y_{n}+v_{n}, \quad a_{Q * P}=a_{Q}+u
$$

and assume that terms of order $u v_{n}, v_{n}^{2}, u^{2}$ may be neglected compared with $v_{n}$ and $u$.

When $y_{n-1} \neq 0$ (or $r \neq 1$ ) the iteration formula (5.1) is rewritten

$$
v_{m k+r}=a_{P} \alpha_{r-2}\left|y_{r-1}\right|^{\epsilon}(1+\epsilon) v_{m k+r-1}-u\left|y_{r-1}\right|^{1+\epsilon}
$$

although, if $y_{n-1}=0$ (i.e. $r=1$ ), $v_{m k+1}=-a_{Q}\left|v_{m k}\right|^{1+\epsilon}$.

The 'first-order' solution of the above recurrence equations is not obvious. After tedious manipulations and using results of $\S 5.1$ we derive the expression for $v_{k l}\left(l^{\prime} \leqslant l\right)$. As $v_{k l}=0$ (periodicity condition) we get

$$
\ln \left[u^{\epsilon}\left|y_{1} \ldots y_{k-1}\right|^{\epsilon}\left(a_{Q}^{k} / a_{P}\right)(1+\epsilon)^{k-1}\left|\left(\mathrm{~d} / \mathrm{d} a_{Q}\right) x_{k}\left(a_{Q}\right)\right|^{\epsilon}\right]=0
$$

whence $u$ and the formula

$$
a_{Q * P} \sim a_{Q}+a_{Q}^{-k / \epsilon} a_{P}^{1 / \epsilon} \mathrm{e}^{-(k-1)} /\left|x_{1} \ldots x_{k-1}\right|\left|\left(\mathrm{d} / \mathrm{d} a_{Q}\right) x_{k}\left(\bar{a}_{Q}\right)\right| .
$$

The correction is small and the displacement is negligible for large periods. 
More generally, to first order

$$
a_{Q^{* n+1} * P}=a_{Q}+a_{Q}^{-k / \epsilon} a_{Q^{1 / n} * P}^{1 / \epsilon} \mathrm{e}^{-(k-1)} /\left|x_{1} \ldots x_{k-1}\right|\left(\mathrm{d} / \mathrm{d} a_{Q}\right) x_{k}\left(\bar{a}_{Q}\right)+\ldots
$$

The limit point $a_{*}$ is the solution of

$$
a_{*}=a_{Q}+a_{Q}^{-k / \epsilon} \mathrm{e}^{-(k-1)} a_{*}^{1 / \epsilon} /\left|x_{1} \ldots x_{k-1}\right|\left(\mathrm{d} / \mathrm{d} a_{Q}\right) x_{k}\left(\bar{a}_{Q}\right)
$$

and, as $a_{Q^{* n} * P}=a_{Q}+\mathrm{O}\left(a_{Q}^{-(k-1) / \epsilon}\right)$,

$$
\left.\delta_{Q}^{-1} \sim \frac{\partial a_{Q^{* n+1} * P}}{\partial a_{Q^{* n} * P}}\right|_{a_{Q}^{* n_{* P} \sim a_{Q}}}
$$

(equation (3.14)), whence

$$
\delta_{Q}^{-1} \sim\left[\mathrm{e}^{-(k-1)} /\left|x_{1} \ldots x_{k-1}\right|\left(\mathrm{d} / \mathrm{d} a_{Q}\right) x_{k}\left(\bar{a}_{Q}\right)\right] \epsilon^{-1} a_{Q}^{-k / \epsilon}\left(a_{*}^{1 / \epsilon}\right) / a_{*}
$$

and both $a_{*}$ and $\delta_{Q}$ do not depend on $P$, as expected. The bifurcation velocity for $Q \neq R$ goes to infinity when $\epsilon \rightarrow 0$, although $\delta_{R} \sim 2$ (see $\S 3.2$ ). Notice that we get the same first-order formula as in $\S 4$.

Expression (5.13) for the bifurcation velocity is a good approximation even when $\epsilon$ is not too small. If we set $\epsilon=1$ and $Q=R L$, then $a_{Q}=1.7549, \bar{a}_{Q}=1.6180$, and we get from (5.13) $\delta_{R L}^{-1}=0.01812$, which is very close to the exact result.

Using now (5.12) for sequences $\left(Q_{1} * Q_{2}\right)^{* n} * P$ and $Q_{2} *\left(Q_{1} * Q_{2}\right)^{* n-1} * P, Q_{1}$ and $Q_{2}$ being primary and $Q_{1}, Q_{2} \neq R$, we get coupled equations for $a_{\left(Q_{1} * Q_{2}\right)^{* n} * P}$ and $a_{Q_{1 *(}\left(Q_{2} * Q_{1}\right)^{*(n-1) * P} \text {, whence the multiplication formula }}$

$$
\delta_{\mathrm{Q}_{1} * \mathrm{Q}_{2}} \sim \delta_{\mathrm{Q}_{1}} \delta_{\mathrm{Q}_{2}}
$$

\subsection{The case $Q=R$}

The derivation of the above formulae does not work in the case $Q=R$, as $a_{R}=1$ and the quantity $a_{Q}^{-k / \epsilon}$ which was considered as small in the previous case is now equal to 1 . One consequence is that the values of superstability for the parameter of the harmonics $R^{* n}$ behave roughly as $1+c_{n} \epsilon \ln \epsilon\left(c_{n}\right.$ all distinct). Another big difference is that the bifurcation velocity is no longer infinite at $\epsilon=0$, since $\delta_{R}=2$ at $\epsilon=0$. We shall see that the corrective term is a very slowly varying function of $\epsilon$ as $\delta_{R} \sim 2+\epsilon /\left(a_{*}-1\right)$, with $a_{*}-1 \sim-\epsilon \ln \epsilon$. For example, when $\epsilon=10^{-3}, \delta_{R}$ is still noticeably larger than its limit value. We find $\delta_{R} \sim 2 \cdot 25$ !

Proof. For a given parameter $a=1+\varphi, \varphi$ being small, the set $\left\{t_{j}(\varphi)\right\}_{j=1, \ldots, 2^{n+1}}$ of the iterates of the maximum satisfy the recurrence relation

$$
t_{2^{n}+p}(\varphi)=t_{p}(\varphi)+\left|\psi_{n}(\varphi)\right|\left(A_{p}+B_{p} \epsilon \ln \left|\psi_{n}(\varphi)\right|+C_{p} \epsilon+D_{p} \varphi\right)
$$

where $1 \leqslant p \leqslant 2^{n}, \psi_{n}(\varphi)=t_{2^{n}}(\varphi)$, and the coefficients $A_{p}, B_{p}, C_{p}$ and $D_{p}$ are to be found by recursion and depend on the $\left(2^{n}-1\right)$ numbers $\alpha_{i}$ defined from the sequence $R^{* n}$ following the rules given in $I$ or in equation $(2.10)$. One has

$$
\begin{aligned}
& A_{p}=B_{p}=-\alpha_{p-2} \ldots \alpha_{0}, \quad D_{p}=p A_{p} \\
& C_{p}=-(p-1) \alpha_{p-2} \ldots \alpha_{0}-\alpha_{p-2} \ldots \alpha_{0} \ln \left|t_{1}(\varphi) \ldots t_{p-1}(\varphi)\right|
\end{aligned}
$$


whence, as $\operatorname{sgn} \psi_{n}=(-1)^{n}$,

$\psi_{n+1}(\varphi)=\psi_{n}(\varphi)\left[\epsilon \ln \left|\psi_{n}(\varphi)\right|+\left(2^{n}-1\right) \epsilon+\epsilon \ln \left|t_{1}(\varphi) \ldots t_{2^{n}-1}(\varphi)\right|+2^{n} \varphi\right]$

for every small $\varphi$.

The value of $\varphi$ for which $R^{*(n+1)}$ is superstable is the solution $\varphi(>0)$ of

$$
\epsilon \ln \left|\psi_{n}(\varphi)\right|+\left(2^{n}-1\right) \epsilon+\epsilon \ln \left|t_{1}(\varphi) \ldots t_{2^{n}-1}(\varphi)\right|+2^{n} \varphi=0
$$

for example, for sequence $R, \psi_{1}(\varphi)=\varphi$ and $\varphi_{1}=0$, and for sequence $R * R, \psi_{2}(\varphi)=$ $\varphi(\epsilon \ln \varphi+\epsilon+2 \varphi)$; and $\varphi_{2}$ is the solution of

$$
\epsilon \ln \varphi+\epsilon+2 \varphi=0, \quad \varphi_{2} \sim-\frac{1}{2} \epsilon(\ln \epsilon+\ln |\ln \epsilon|+1-\ln 2) .
$$

The calculations quickly become very tedious in this way, and we substitute for sequence $\psi_{n}(\varphi)$ the sequence $\chi_{n+1}(\varphi)=\psi_{n+1}(\varphi) / \psi_{n}(\varphi)\left[\chi_{1}=\varphi\right]$. It is easy to prove that

$$
\chi_{n+1}(\varphi)=2 \chi_{n}(\varphi)+\epsilon+\epsilon \ln \chi_{n}(\varphi)
$$

(we use $\ln \left|t_{1}(\varphi) \ldots t_{2^{n+1}-1}(\varphi)\right| \sim 2 \ln \left|t_{1}(\varphi) \ldots t_{2^{n}-1}(\varphi)\right|+\ln \left|\psi_{n}(\varphi)\right|$ ) and $\varphi_{n}$ is the root of $\chi_{n}\left(\varphi_{n}\right)=0$.

As $\chi_{n}\left(\varphi_{n}\right)=\chi_{n+1}\left(\varphi_{n+1}\right)=0$, we get from (5.16)

$\chi_{n-1}\left(\varphi_{n}\right)=\chi_{n}\left(\varphi_{n+1}\right), \quad \chi_{n-2}\left(\varphi_{n}\right)=\chi_{n-1}\left(\varphi_{n+1}\right) \ldots \chi_{1}\left(\varphi_{n}\right)=\varphi_{n}=\chi_{2}\left(\varphi_{n+1}\right)$

whence

$$
2 \varphi_{n+1}+\epsilon+\epsilon \ln \varphi_{n+1}=\varphi_{n} .
$$

The limit value $\varphi_{*}=a_{*}-1$ is then the solution of

$$
\varphi_{*}+\epsilon+\epsilon \ln \varphi_{*}=0
$$

and

$$
\begin{aligned}
& \delta_{R}=\lim _{n \rightarrow \infty}\left(\partial \varphi_{n} / \partial \varphi_{n+1}\right)=\lim _{n \rightarrow \infty}\left(2+\epsilon / \varphi_{n+1}\right) \\
& \delta_{R}=2+\epsilon / \varphi_{*}
\end{aligned}
$$

which is equation (4.10).

Notice that for $\epsilon=1, \varphi_{*} \sim 0 \cdot 28$, and using formula (5.19) we get $\delta_{R} \sim 5 \cdot 57$ which is not so bad as the relative error is less than $20 \%$.

\section{Conclusion}

In I, the authors showed that the whole set of sequences of Metropolis, Stein and Stein has a property of internal similarity; in the present paper, it is shown that this internal similarity is not only of a purely algebraic nature. After a large number of applications of one of these similarity transformations, one gets a transform which is actually a contraction in the parameter space, with a constant ratio that is independent of the general shape of the transform, but depends only on its analytic structure near the maximum.

It is also rather striking to observe that this kind of property exists also in the case of invertible mapping, as encountered in dynamical systems. In particular we have studied 
from this point of view the Hénon two-dimensional mapping $T_{a, b}(x, y) \rightarrow$ $\left(1-a x^{2}+y, b x\right), a$ and $b$ being parameters, and $x$ and $y$ real quantities (Hénon 1976).

There is no longer a critical point, as this mapping is invertible and multiplies the area by a constant factor $b$. Choosing for $b$ the same value as Hénon did, $b=0 \cdot 3$, it is possible to follow on a computer the bifurcation from a stable period 1 to a stable period 2 , to a stable period $4 \ldots$ to a stable period $2^{n}$. The accumulation point for this set of bifurcations is reached at $a_{*} \sim 1.058048 \ldots$ when $b=0.3$. In table 5 we give the values of $a$ corresponding to the occurrence of a stable period $2^{n}$ with $0 \leqslant n \leqslant 8$ (and to the destabilisation of the period $2^{n-1}$ ). The computations are noticeably more lengthy than in the case of endomorphisms of the line, as it is not possible to use the Newton method for finding the point of superstability which does not exist in this case, as the Hénon mapping has a constant Jacobian. It is rather striking to observe that all these values of $a$ approach the limit $a_{*}$ in a geometric fashion, and that the bifurcation velocity is the same (up to three digits) as in the case of $C^{\infty}$ endomorphisms of the line. In order to justify this fact (in a very sloppy way of course!), one might just observe that after a large number of iterations, say $N$, the elementary area is multiplied by $b^{N}$ and thus shrinks to zero, as $N \rightarrow \infty$ when $b<1$, so that the mapping is, in some sense, 'one-dimensional'. We hope to come to this point in a future work.

Table 5. List of the zones of stability and approximations for the sequences $R^{* n}$ in the Hénon mapping. The calculations correspond to $b=0 \cdot 3$. The accuracy of $\delta$ increases with $n$. We use formula (3.5) for $\delta(n)$.

\begin{tabular}{llll}
\hline$n$ & Period, $2^{n}$ & $\begin{array}{l}a_{\mathbf{s}} \text { (beginning } \\
\text { of stability) }\end{array}$ & $\begin{array}{l}\text { Approximation } \\
\text { for } \delta\end{array}$ \\
\hline 0 & 1 & $\begin{array}{l}-(1-b)^{2} / 4=-0.1225 \\
3(1-b)^{2} / 4=0.3675\end{array}$ \\
1 & 2 & $\frac{5+5 b^{2}-6 b}{4}=0.9125$ & 4.844 \\
2 & 4 & 1.026 & 4.3269 \\
3 & 8 & 1.051 & 4.696 \\
4 & 16 & 1.056536 & 4.636 \\
5 & 32 & 1.05773083 & 4.7748 \\
6 & 64 & 1.0579808931 & 4.6696 \\
7 & 128 & 1.05803445215 & 4.6691 \\
8 & 256 & 1.05804592304 & 4.6691 \\
9 & 512 & 1.05804837980 & 4.6694 \\
10 & 1024 & 1.058048905931 & \\
11 & 2048 & & \\
\hline
\end{tabular}

\section{Acknowledgment}

The authors are grateful to Dr T W Burkhardt for many useful discussions on the comparison with phase transition problems.

\section{References}

Cosnard M Y and Eberhard A 1977 Séminaire Analyse Numérique No. 274 Lab. Math. Appl., USMG, Grenoble 
Derrida B, Gervois A and Pomeau Y 1977 C.R. Acad. Sci., Paris A 28543 1978 Ann. Inst. Henri Poincaré 29305

Derrida B and Pomeau Y 1979 Preprint

Domb C and Green M S 1976 Phase Transitions and Critical Phenomena Vol 6 (New York: Academic) Feigenbaum M J 1977 a Proc. Como Conf. on Stochasticity of Hamiltonian systems ed. J Ford 1977 b SIAM J.

Guckenheimer J 1977 Invent. Math. 39165

Hénon M 1976 Commun. Math. Phys. 5069

Li T Y and Yorke J A 1975 Am. Math. Mon. 82985

1979 Trans. Am. Math. Soc. in the press

May R M 1976 Nature 261459

Metropolis N, Stein M L and Stein P R 1973 J. Comb. Theory A 1525

Sarkovskii A N 1964 Ukr. Math. J. 1661

Stefan P 1977 Commun. Math. Phys. 54237 